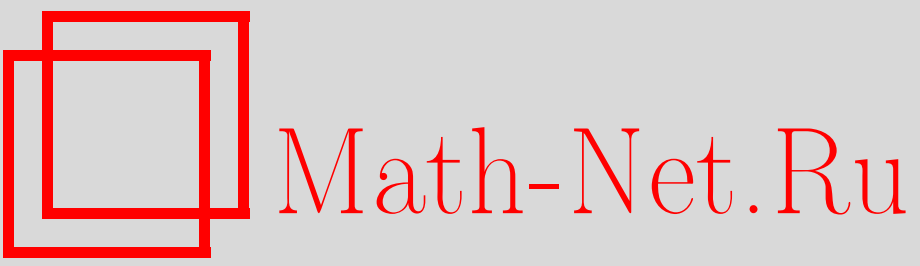

И. А. Баталин, А. М. Семихатов, Ассоциативность и операторное гамильтоново квантование калибровочных теорий, ТМФ, 2003, том 136, номер 3, 339-364

DOI: https://doi.org/10.4213/tmf229

Использование Общероссийского математического портала Math-Net.Ru подразумевает, что вы прочитали и согласны с пользовательским соглашением

http://www.mathnet.ru/rus/agreement

Параметры загрузки:

IP: 54.224 .60 .19

26 апреля 2023 г., 06:32:17

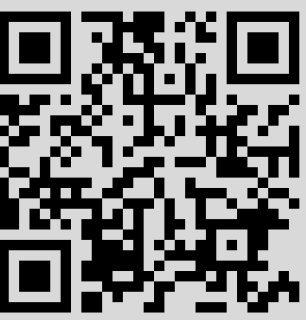




\author{
ТЕОРЕТИЧЕСКАЯ \\ И МАТЕМАТИЧЕСКАЯ \\ ФИЗИКА \\ Том 136, № 3 \\ сентябрь, 2003
}

(C) 2003 г.

И. А. Баталин ${ }^{*}$, А. М. Семихатов*

\title{
АССОЦИАТИВНОСТЬ И ОПЕРАТОРНОЕ ГАМИЛЬТОНОВО КВАНТОВАНИЕ КАЛИБРОВОЧНЫХ ТЕОРИЙ
}

Показано, что формализм БРСТ-квантования калибровочных теорий можно распространить на структуру ассоциативной алгебры, причем переход от соответствующей алгебры Ли к ассоциативной алгебре достигается с использованием операторного квантования приводимых калибровочных теорий. БРСТ-дифференциал, кодирующий ассоциативность умножения в алгебре, построен как квадратичный дифференциальный оператор второго порядка на bar-резольвенте.

Ключевые слова: ассоциативные алгебры, БРСТ-дифференциал, bar-резольвента, некоммутативные дифференциальные формы.

\section{1. ВВЕДЕНИЕ}

Методы БРСТ-квантования систем со связями (или, что то же, калибровочных теорий) [1]-[3] имеют репутацию эффективного подхода, выходящего за рамки той области, в которой эти методы были исходно созданы. В гамильтоновом формализме БРСТквантование системы со связями первого рода сводится к построению нечетного дифференциала ("БРСТ"-оператора) в системе, расширенной духами и сопряженными импульсами (на классическом уровне являюшимися переменными Кошуля-Тейта [4]). Однако формализм ограничен алгебраическими структурами, построенными на основе (градуированно) антисимметричных операций (порожденных коммутаторами или скобками Пуассона).

В данной работе мы показываем, что гамильтонов БРСТ-формализм применим также и к градуированным ассочиативныц алгебрам, а потому не ограничен Ли-подобными структурами. Для заданной ассоциативной алгебры $\mathcal{A}$ мы построим такой дифоференциал $\boldsymbol{\Omega}$, что соотношение $\boldsymbol{\Omega}^{2}=0$ является "БРСТ-перекодировкой" ассоциативности умножения в $\mathcal{A}$.

Конструкция содержит "тривиальную” часть - ассоциированную (градуированную) алгебру Ли $(\mathcal{A},[]$,$) , которая обрабатывается стандартными БРСТ-методами, и “слож-$ ную" часть, состояшую в распространении БРСТ-схемы на ассоциативную алгебру с использованием методов приводимых калибровочных теорий [5] таким образом, что

* Физический институт им. П. Н. Лебедева РАН, Москва, Россия. E-mail: ams@sci.lebedev.ru 
bar-резольвента алгебры $\mathcal{A}$ рассматривается как набор данных, определяющих приводимую калибровочную теорию. В соответствии с БРСТ-идеологией мы вводим духи для каждого члена bar-резольвенты, квантуем их и ищем БРСТ-дифференциал $\boldsymbol{\Omega}$ с духовым числом 1 ; мы также требуем, чтобы $\Omega$ был не более чем квадратичным по духам и не более чем билинейным по импульсам, и чтобы ни один импульс не входил в $\Omega$ квадратично. БРСТ-дифференциал является, таким образом, квадратичным дифференциальным оператором второго порядка на bаr-резольвенте; более того, его квадратичная часть является оператором со скалярными коэффициентами. В точном виде это утверждение будет сформулировано ниже (см. п. 3.4).

Хотя наша конструкция дифференциала $\boldsymbol{\Omega}$ мотивирована БРСТ-методами, соответствие с известным БРСТ-формализмом для приводимых калибровочных теорий несколько ограничено, поскольку bar-резольвента бесконечна, а потому соответствуюшая приводимая калибровочная теория имеет бесконечную степень приводимости. Несмотря на определенные усилия [5], [6], дополнительно мотивированные возможными применениями в теории струн [7], бесконечно-приводимые калибровочные теории не получили законченной формулировки, позволяюшей доказать сушествование БРСТ-дифференциала (или решения мастер-уравнения в лагранжевой формулировке). Стандартный индукционный аргумент, применимый к конечно-приводимым калибровочным теориям, не работает в бесконечно-приводимом случае, и поэтому нам придется дать независимое доказательство для ассоциативных алгебр; мы построим рекурсионную процедуру, которая приводит к решению уравнения $\Omega^{2}=0$. Это позволит также обойти другое усложнение: в отличие от "настояших" калибровочных теорий, где связи первого рода удовлетворяют так называемым соотношениям инволюции (преврашаюшимся в соотношения в терминах скобок Пуассона при $\hbar \rightarrow 0$ )

$$
\left[T_{\alpha}, T_{\beta}\right]=i \hbar \sum_{\gamma} U_{\alpha \beta}^{\gamma} T_{\gamma},
$$

в которых $U_{\alpha \beta}^{\gamma}$ - некоторые операторы, коммутаторы в $\mathcal{A}$ не содержат постоянной Планка $\hbar$. Другими словами, постоянная Планка в предлагаемом формализме равна 1 (точнее, $-i)$, а потому у соответствуюшей калибровочной теории нет классического предела, и БРСТ-формализм следует применять непосредственно на операторном уровне.

Данная работа построена следуюшим образом. В разделе 2 мы напоминаем основные факты о приводимых калибровочных теориях. В п. 3.1 объясняем соотношение между разделом 2 и основной частью раздела 3 . В п. 3.3 мы вводим духи и строим дифференциал как БРСТ-оператор в специальной (бесконечно) приводимой калибровочной теории. Основной результат о том, что $\boldsymbol{\Omega}^{2}=0$, сформулирован в п. 3.4 и доказан в п. $3.5-3.7$.

\section{2. ПРИВОДИМЫЕ СИСТЕМЫ СО СВЯЗЯМИ}

В данном разделе суммированы основные положения гамильтонова БРСТ-квантования систем со связями, используемые для мотивировки дальнейших построений. Связь меж ду БРСТ-формализмом и нашей основной конструкцией будет объяснена в п. 3.1.

2.1. Классические приводимые системы со связями. Классическая приводимая система со связями [5] состоит из 
- симплектического многообразия $\mathcal{X}$ (в действительности - симплектического векторного пространства) и (достаточно гладких) функций $T_{\alpha_{0}}, \alpha_{0} \in \mathcal{J}_{0}$, на $\mathcal{X}$, называемых связями, нулевой локус которых называется "поверхностью" связей $\mathcal{S} \subset \mathcal{X}$ и рассматривается физиками как нечто очень близкое к гладкому многообразию;

- функций $Z_{\alpha_{n}}^{\alpha_{n-1}}, \alpha_{i} \in \mathcal{J}_{i}$, на $\mathcal{X}$, удовлетворяющих ранговым условиям и уравнениям “нулевых мод" (2.1) и (2.2), приведенным ниже.

Рассмотрим эти составляюшие подробнее. Если связи линейно независимы над $C^{\infty}(\mathcal{X})$, теория называется неприводимой; в противном случае существуют функции $\stackrel{1}{Z}_{\alpha_{1}}^{\alpha_{0}}, \alpha_{1} \in \mathcal{J}_{1}$, на $X$ такие, что

$$
\sum_{\alpha_{0} \in \mathcal{J}_{0}}{ }_{Z}^{\alpha_{\alpha_{1}}} T_{\alpha_{0}}=0
$$

Функции $\stackrel{1}{Z}_{\alpha_{1}}^{\alpha_{0}}$, в свою очередь, могут быть линейно зависимыми над $C^{\infty}(\mathcal{X})$, что вводит в рассмотрение некоторые функции $\stackrel{2}{Z}_{\alpha_{2}}^{\alpha_{1}}$, и т.д.; точнее, каждая последующая линейная зависимость требуется только в "слабой” форме, т.е. по модулю идеала J, порожденного связями $\left\{T_{\alpha_{0}}\right\}$ :

$$
\sum_{\alpha_{n-1}}{\stackrel{n}{Z_{n}}}_{\alpha_{n-1}}^{n-1}{ }^{\alpha_{\alpha}}{ }_{\alpha_{n-1}}^{\alpha_{n-2}} \in \mathcal{J}, \quad \alpha_{i} \in \mathcal{J}_{i}, \quad n \geqslant 2
$$

(мы будем иногда опускать верхнее $n$ в обозначениях). При ограничении на поверхность связей это дает комплекс, который является по определению точным. Таким образом, если рассматривать каждую $Z_{\alpha_{n}}^{\alpha_{n-1}}$ как прямоугольную матрицу, ранги их ограничений на $\mathcal{S}$ должны удовлетворять условиям

$$
\begin{aligned}
& \left.\operatorname{rank}\left(\stackrel{n+1}{Z} \underset{\alpha_{n+1}}{\alpha_{n}}\right)\right|_{\mathcal{S}}+\left.\operatorname{rank}\left(Z_{Z_{n}}^{\alpha_{n-1}}\right)\right|_{\mathcal{S}}=\operatorname{card}_{n}, \quad n \geqslant 1, \\
& \left.\operatorname{rank}\left(Z_{\alpha_{1}}^{\alpha_{0}}\right)\right|_{\mathcal{S}}+\left.\operatorname{rank}\left(\frac{\partial T_{\alpha_{0}}}{\partial x^{i}}\right)\right|_{\mathcal{S}}=\operatorname{card} \mathcal{J}_{0}
\end{aligned}
$$

где $\left\{x^{i}\right\}$ - любая локальная система координат в окрестности $\mathcal{S}$ в $\mathcal{X}$. Эти ранги предполагаются постоянными в некоторой окрестности $\mathcal{S}$ в $\mathcal{X}$ [3], [5]. Мы будем говорить о $\stackrel{n}{Z}$ как о "нулевых модах." Введем также $\mathbb{Z}_{2}$-градуировки

$$
\varepsilon\left(Z_{\alpha_{n}}^{\alpha_{n-1}}\right)=\varepsilon_{\alpha_{n}}+\varepsilon_{\alpha_{n-1}}, \quad \varepsilon\left(T_{\alpha_{0}}\right)=\varepsilon_{\alpha_{0}} .
$$

ОПРЕДЕЛЕНИЕ 2.1. Система со связями называется $\ell$-приводимой, если $\stackrel{\ell+1}{Z}=0$ и $\stackrel{\ell}{Z} \not \equiv 0$. В частности, 0-приводимая теория является неприводимой (соотношения (2.1) отсутствуют).

Систему со связями можно расширить вспомогательными переменными, называемыми духами и сопряженными импульсами, так, чтобы существовало нечетное гамильтоново векторное поле $\{\Omega,-\}$ (БРСТ-оператор), квадрат которого равен нулю, а низшие члены в разложении $\Omega$ по духам включают связи и нулевые моды $\stackrel{n}{Z}$. Во избежание повторов рассмотрим подробности сразу в квантовой ситуации. 
2.2. Квантовая приводимая система со связями. Математическое существование квантовой теории является деликатным вопросом, и следующие утверждения, строго говоря, чувствительны к выбранному квантованию. Деформационное квантование [8] само по себе не позволяет автоматически говорить об операторных соотношениях, однако мы будем их использовать при формулировке основных положений, составляющих фольклор калибровочных теорий.

2.2.1. На квантово-механическом уровне $T_{\alpha_{0}}$ и $Z_{\alpha_{n}}^{\alpha_{n-1}}$ становятся операторами из некоторой алгебры $\mathbb{A}^{\hbar}$. Уравнение $(2.1)$ при этом сохраняет свой вид в терминах элементов $\mathbb{A}^{\hbar}$, а соотношения (2.2) принимают следуюший вид:

$$
\sum_{\alpha_{n-1}} \stackrel{n}{Z}_{\alpha_{n}}^{\alpha_{n-1}}{ }^{n-1}{ }^{\alpha_{n-2}}{ }_{\alpha_{n-1}}^{\alpha_{n-1}}=\sum_{\alpha_{0}} \Pi_{\alpha_{n}}^{\alpha_{n-2} \alpha_{0}} T_{\alpha_{0}}+\hbar \mathbb{A}^{\hbar}, \quad \alpha_{i} \in \mathcal{J}_{i}
$$

где $\Pi_{\alpha_{n}}^{\alpha_{n-2} \alpha_{0}} \in \mathbb{A}^{\hbar}$.

2.2.2. Состав духов. Алгебра $\mathbb{A}^{\hbar}$ расширяется до $\mathbb{A}_{\mathrm{gh}}^{\hbar}$ множеством операторов $\left\{C^{A}\right\}$ (духов) и $\left\{\overline{\mathcal{P}}_{A}\right\}$ (сопряженных импульсов), удовлетворяющих каноническим градуированным коммутационным соотношениям $\left[\overline{\mathcal{P}}_{B}, C^{A}\right] \equiv \overline{\mathcal{P}}_{B} C^{A}-(-1)^{\varepsilon\left(\overline{\mathcal{P}}_{B}\right) \varepsilon\left(C^{A}\right)} C^{A \overline{\mathcal{P}}_{B}}=$ $i \hbar \delta_{B}^{A}$. Здесь $A$ - набор (мульти)индексов, $A=\left\{\alpha_{0}, \alpha_{1}, \ldots, \alpha_{\ell}\right\}, \alpha_{i} \in \mathcal{J}_{i}$, так что духи представляют собой набор $\left\{C^{A}\right\}=\left\{C^{\alpha_{0}}, \ldots, C^{\alpha_{\ell}}\right\}, \alpha_{i} \in \mathcal{J}_{i}$, и, аналогично, для импульсов $\left\{\overline{\mathcal{P}}_{B}\right\}=\left\{\overline{\mathcal{P}}_{\beta_{0}}, \ldots, \overline{\mathcal{P}}_{\beta_{\ell}}\right\}, \beta_{i} \in \mathcal{J}_{i} ; \mathbb{Z}_{2}$-градуировки духов и импульсов равны $\varepsilon\left(C^{\alpha_{n}}\right)=\varepsilon\left(\overline{\mathcal{P}}_{\alpha_{n}}\right)=\varepsilon_{\alpha_{n}}+n+1 ;$ духовые числа приписываются духам и импульсам как $\operatorname{gh} C^{\alpha_{n}}=n+1$ и gh $\overline{\mathcal{P}}_{\alpha_{n}}=-n-1$.

2.2.3. Следующее утверждение представляет собой основное рабочее средство в гамильтоновом квантовании систем со связями (см. замечания ниже).

ТЕорема 2.1 (Баталин-Фрадкин). В квантовой $\ell$-приводимой системе со связями существует 2-нильпотентный нечетный оператор $\Omega$ с духовым числом 1 (БРСТ-дифференциал), $\varepsilon(\Omega)=1, \operatorname{gh} \Omega=1, \Omega^{2}=0$, вида

$$
\Omega=\sum_{n \geqslant 1} \sum_{m \geqslant 0} \sum_{\substack{A_{1}, \ldots, A_{m} \\ B_{1}, \ldots, B_{n}}} C^{A_{m}} \ldots C^{A_{1}} V_{A_{1} \ldots A_{m}}^{B_{n} \ldots B_{1}} \overline{\mathcal{P}}_{B_{1}} \ldots \overline{\mathcal{P}}_{B_{n}} \in \mathbb{A}_{\mathrm{gh}}^{\hbar}
$$

где $V_{A_{1} \ldots A_{m}}^{B_{n} \ldots B_{1}}-$ onepamopbl из $\mathbb{A}^{\hbar}$ maкue, что

$$
V_{\alpha_{0}}=T_{\alpha_{0}}, \quad V_{\alpha_{n}}^{\alpha_{n-1}}=\stackrel{n}{Z}_{\alpha_{n}}^{\alpha_{n-1}},
$$

и при $\hbar \rightarrow 0$ операторь $T_{\alpha_{0}}$ и $\stackrel{n}{Z}_{\alpha_{n}}^{\alpha_{n-1}}$ становятся соответствующими данными, определяющими классическую $\ell$-приводимую калибровочную теорию, а $\Omega$ становится нечетной функиией, удовлетворяющей соотношению $\{\Omega, \Omega\}=0$ в терминах скобки Пуассона (скобка Пуассона между духами и импульсами определяется их коммутатором). 
ЗАмечАниЕ 2.1. В теореме утверждается сушествование операторов $V_{A_{1} \ldots A_{m}}^{B_{n} \ldots B_{1}}$ помимо тех, что фиксированы “граничными условиями” (2.6). Другими словами, при заданных связях и операторах $\stackrel{i}{Z}$ можно построить нильпотентный $\Omega$ с духовым числом 1 в виде формального ряда по духам и импульсам, начиная со слагаемых низшего порядка:

$$
\Omega=\sum_{\alpha_{0}} C^{\alpha_{0}} T_{\alpha_{0}}+\sum_{n=1}^{\ell} \sum_{\alpha_{n}, \alpha_{n-1}} C^{\alpha_{n}} \stackrel{n}{Z}_{\alpha_{n}}^{\alpha_{n-1}} \overline{\mathcal{P}}_{\alpha_{n-1}}+\cdots
$$

Однако существование квантового БРСТ-оператора в виде ряда по духам никогда не доказывалось явно. Известно, что его классический аналог существует, если $\mathcal{X}$ является симплектическим линейным пространством и гомологии оператора Кошуля-Тейта сконцентрированы в нулевом духовом числе (чем и определяется значение предположений о рангах). Классический БРСТ-оператор можно тогда подвергнуть деформационному квантованию, о сушествовании которого на языке символов (в виде формального ряда по $\hbar)$ можно говорить, если классический оператор $\{\Omega,-\}$ имеет тривиальные гомологии в духовом числе 1 .

ЗАмЕчАнИЕ 2.2. Коэффициенты в (2.5) традиционно переопределяются как

$$
V_{A_{1} \ldots A_{m}}^{B_{n} \ldots B_{1}}=(-1)^{E_{A_{1} \ldots A_{m}}^{B_{n} \ldots B_{1}}} \frac{1}{m ! n !} U_{A_{1} \ldots A_{m}}^{B_{n} \ldots B_{1}}
$$

где знаковые множители ([] - целая часть)

$$
\begin{aligned}
E_{A_{1} \ldots A_{m}}^{B_{n} \ldots B_{1}}= & \sum_{k=1}^{[m / 2]} \varepsilon\left(C^{A_{2 k}}\right)+\sum_{k=1}^{[n / 2]} \varepsilon\left(\overline{\mathcal{P}}_{B_{2 k}}\right)+ \\
& +\left[\frac{m}{2}\right] \max \operatorname{gh}\left(C^{A_{1}}, \ldots, C^{A_{m}}\right)+\left[\frac{n}{2}\right] \max \operatorname{gh}\left(\overline{\mathcal{P}}_{B_{1}}, \ldots, \overline{\mathcal{P}}_{B_{n}}\right)
\end{aligned}
$$

выбраны таким образом, чтобы минимизировать появление знаковых множителей в переписанных через $U_{A_{1} \ldots A_{m}}^{B_{n} \ldots B_{1}}$ соотношениях, порожденных равенствами $(1.1)$ и соотношениями приводимости; знаковые множители тогда возникают только в суммах по циклическим перестановкам.

ЗАмЕчАниЕ 2.3. Ниже будет изучаться специальный случай, когда $\varepsilon_{\alpha_{i}}=0$, и тем самым $\mathbb{Z}_{2}$-градуировки духов равны $\varepsilon\left(C^{\alpha_{i}}\right)=i+1, i=0, \ldots, \ell$, а все $U_{A_{1} \ldots A_{m}}^{B_{n} \ldots B_{1}}$ являются четными: $\varepsilon\left(U_{A_{1} \ldots A_{m}}^{B_{n} \ldots B_{1}}\right)=0$. Младшие уравнения, следуюшие из $\Omega \Omega=0$, даются соотношениями инволюции (1.1), условиями "нулевых мод" (2.1) и

$$
\begin{aligned}
& (i \hbar)^{-1}\left[T_{\alpha_{0}}, Z_{\alpha_{1}}^{\beta_{0}}\right]-Z_{\alpha_{1}}^{\gamma_{0}} U_{\gamma_{0} \alpha_{0}}^{\beta_{0}}=-U_{\alpha_{0} \alpha_{1}}^{\gamma_{0} \delta_{0}} \Pi_{\gamma_{0} \delta_{0}}^{\beta_{0}}-U_{\alpha_{0} \alpha_{1}}^{\beta_{1}} Z_{\beta_{1}}^{\beta_{0}}, \\
& 2 \Pi_{\alpha_{0} \beta_{0}}^{\gamma_{0}}=T_{\left[\alpha_{0}\right.} \delta_{\left.\beta_{0}\right]}^{\gamma_{0}}-i \hbar U_{\alpha_{0} \beta_{0}}^{\gamma_{0}}, \\
& Z_{\alpha_{2}}^{\alpha_{1}} Z_{\alpha_{1}}^{\alpha_{0}}=-U_{\alpha_{2}}^{\beta_{0} \gamma_{0}} \Pi_{\beta_{0} \gamma_{0}}^{\alpha_{0}} \\
& (i \hbar)^{-1}\left[Z_{\alpha_{1}}^{\left[\alpha_{0}\right.}, Z_{\beta_{1}}^{\left.\beta_{0}\right]}\right]-Z_{\left\{\alpha_{1}\right.}^{\gamma_{0}} U_{\left.\gamma_{0} \beta_{1}\right\}}^{\alpha_{0} \beta_{0}}= \\
& =-U_{\alpha_{1} \beta_{1}}^{\left[\alpha_{0} \gamma_{0} \delta_{0}\right.} \Pi_{\gamma_{0} \delta_{0}}^{\left.\beta_{0}\right]}-U_{\alpha_{1} \beta_{1}}^{\left[\alpha_{0} \gamma_{1}\right.} Z_{\gamma_{1}}^{\left.\beta_{0}\right]}+U_{\alpha_{1} \beta_{1}}^{\alpha_{2}} U_{\alpha_{2}}^{\alpha_{0} \beta_{0}}+i \hbar U_{\alpha_{1} \beta_{1}}^{\gamma_{0} \gamma_{1}} U_{\gamma_{0} \gamma_{1}}^{\alpha_{0} \beta_{0}}, \\
& Z_{\left\{\alpha_{1}\right.}^{\alpha_{0}} U_{\left.\alpha_{0} \beta_{1}\right\}}^{\gamma_{1}}+U_{\alpha_{1} \beta_{1}}^{\alpha_{2}} Z_{\alpha_{2}}^{\gamma_{1}}=-U_{\alpha_{1} \beta_{1}}^{\alpha_{0} \delta_{1}}\left(T_{\alpha_{0}} \delta_{\delta_{1}}^{\gamma_{1}}+i \hbar U_{\alpha_{0} \delta_{1}}^{\gamma_{1}}\right)
\end{aligned}
$$


где подразумевается суммирование по повторяюшимся индексам, $A^{[\alpha \beta]}=A^{\alpha \beta}-A^{\beta \alpha}$ и $A^{\{\alpha \beta\}}=A^{\alpha \beta}+A^{\beta \alpha}$; при заключении группы индексов в скобки операции антисимметризации и симметризации применяются только к самому левому и самому правому индексам.

\section{3. Приводимые теории с замкнутой алгеброй.}

ОПРЕДЕЛЕНИЕ 2.2. Будем говорить, что связи первого рода $T_{\alpha_{0}}, \alpha_{0} \in \mathcal{J}_{0}$, порождают замкнутую калибровочную алгебру, если БРСТ-дифференциал $\Omega$ не более чем квадратичен по духам.

"Калибровочная теория", которая будет рассмотрена в разделе 3 , имеет замкнутую калибровочную алгебру, но является бесконечно-приводимой, в силу чего приходится рассматривать суммы по бесконечно большому числу духов. Мы используем индексы $A, B, \ldots$ для нумерации всех духов (т.е. $C^{\alpha_{0}}, C^{\alpha_{1}}, \ldots$ в терминах п. 2.2.2). В теории с замкнутой алгеброй БРСТ-дифференциал можно записать как

$$
\Omega=\sum_{A} C^{A} \tau_{A}+\frac{1}{2} \sum_{A, B}(-1)^{\varepsilon\left(C^{B}\right)+1} C^{B} C^{A} U_{A B}
$$

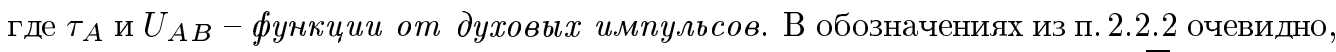
что $\tau_{\alpha_{0}}=T_{\alpha_{0}}$ являются исходными связями; все $\tau_{\alpha_{n}}$ при $n \geqslant 1$ зависят от $\overline{\mathcal{P}}_{C}$. Уравнение $\Omega^{2}=0$ тогда сводится к независимым условиям обращения в нуль для членов первой, второй и третьей степени по $C^{A}$. Предвосхищая полученную в разделе 3 структуру БРСТ-дифференциала (где $U_{A B}$ скалярнозначны), мы выпишем эти уравнения для случая

$$
\left[U_{A B}, U_{C D}\right]=0, \quad\left[\tau_{A}, U_{B C}\right]=0 .
$$

В линейном, квадратичном и кубичном порядках по $C^{A}$ соответствующие уравнения имеют вид (с подразумеваемым суммированием по повторяюшимся индексам)

$$
\begin{gathered}
{\left[\tau_{A}, C^{B}\right] \tau_{B}+\frac{1}{2}\left[\left[\tau_{A}, C^{C}\right], C^{B}\right] U_{B C}(-1)^{\varepsilon\left(C^{A}\right)+1}=0} \\
{\left[\tau_{A}, \tau_{B}\right]=\left[U_{A B}, C^{C}\right] \tau_{C}-\left[\tau_{A}, C^{C}\right] U_{C B}(-1)^{\varepsilon\left(C^{B}\right)+1}+} \\
+\left[\tau_{B}, C^{C}\right] U_{C A}(-1)^{\left(\varepsilon\left(C^{A}\right)+1\right) \varepsilon\left(C^{B}\right)}+\frac{1}{2}\left[\left[U_{A B}, C^{D}\right], C^{C}\right] U_{C D}(-1)^{\varepsilon\left(C^{D}\right)+1} \\
{\left[U_{A B}, C^{D}\right] U_{D C}(-1)^{\left(\varepsilon\left(C^{A}\right)+1\right)\left(\varepsilon\left(C^{C}\right)+1\right)}+\operatorname{cycle}(A, B, C)=0 .}
\end{gathered}
$$

\section{3. БРСТ-ДИФЕРЕНЦИАЛ}

В данном разделе мы построим БРСТ-дифференциал $\boldsymbol{\Omega}$ как дифференциальный оператор на bar-резольвенте заданной ассоциативной алгебры $\mathcal{A}$. Основной результат о том, что $\boldsymbol{\Omega}$ является дифференциалом, будет сформулирован в п. 3.4 ; рекурсионное решение уравнения $\boldsymbol{\Omega} \boldsymbol{\Omega}=0$ построено в п. 3.5 , а доказательство равенства $\boldsymbol{\Omega} \boldsymbol{\Omega}=0$ продолжается до п. 3.7 . 
Пусть $\mathcal{A}$ - ассоциативная градуированная алгебра с единицей. Будем писать $\mathrm{m}_{1, i}$ : $\mathcal{A}^{\otimes n} \rightarrow \mathcal{A}^{\otimes(n-1)}$ для операции умножения в первом сомножителе:

$$
\mathrm{m}_{1, i}: a_{1} \otimes \cdots \otimes a_{n} \mapsto(-1)^{\varepsilon\left(a_{i}\right)\left(\varepsilon\left(a_{2}\right)+\cdots+\varepsilon\left(a_{i-1}\right)\right)} a_{1} a_{i} \otimes a_{2} \otimes \cdots \otimes \not \mu_{i} \otimes \cdots \otimes a_{n}
$$

при $2 \leqslant i \leqslant n$, и используем несколько избыточное обозначение $\mathrm{m}_{i, 1}^{*}$ ( с переставленными нижними индексами и со звездочкой) для умножения в противоположном порядке (но, как и выше, помешенного в первый тензорный множитель):

$$
\mathrm{m}_{i, 1}^{*}: a_{1} \otimes \cdots \otimes a_{n} \mapsto(-1)^{\varepsilon\left(a_{i}\right)\left(\varepsilon\left(a_{1}\right)+\cdots+\varepsilon\left(a_{i-1}\right)\right)} a_{i} a_{1} \otimes a_{2} \otimes \cdots \otimes \not d \otimes \cdots \otimes a_{n} .
$$

Нам также понадобятся обозначения

$$
\operatorname{ad}_{a^{\prime}} \cdot a_{0} \otimes \cdots \otimes a_{n}=\left[a^{\prime}, a_{0}\right] \otimes a_{1} \otimes \cdots \otimes a_{n} .
$$

Будем использовать обозначение s для отображения "правого сдвига" s: $\mathcal{A}^{\otimes n} \rightarrow$ $\mathcal{A}^{\otimes(n+1)}$,

$$
\mathrm{s}: a_{1} \otimes \cdots \otimes a_{n} \mapsto 1 \otimes a_{1} \otimes \cdots \otimes a_{n} .
$$

Наконец, нам понадобится операция передвижения $i$-го тензорного множителя на первую позицию

$$
\mathrm{P}_{1, i}: a_{1} \otimes \cdots \otimes a_{n} \mapsto(-1)^{\varepsilon\left(a_{i}\right)\left(\varepsilon\left(a_{1}\right)+\cdots+\varepsilon\left(a_{i-1}\right)\right)} a_{i} \otimes a_{1} \otimes a_{2} \otimes \cdots \otimes \not q_{i} \otimes \cdots \otimes a_{n}
$$

и обратная операция

$$
\mathrm{P}_{i, 1}: a_{1} \otimes \cdots \otimes a_{n} \mapsto(-1)^{\varepsilon\left(a_{1}\right)\left(\varepsilon\left(a_{2}\right)+\cdots+\varepsilon\left(a_{i}\right)\right)} a_{2} \otimes \cdots \otimes a_{i} \otimes a_{1} \otimes a_{i+1} \otimes \cdots \otimes a_{n} .
$$

3.1. Вar-резольвента и "нулевые моды". Рассмотрим bar-резольвенту алгебры $\mathcal{A}$, состояшую из свободных $\mathcal{A}$-бимодулей

$$
\cdots \stackrel{\mathbf{b}^{\prime}}{\rightarrow} \mathcal{A} \otimes \overline{\mathcal{A}}^{\otimes 2} \otimes \mathcal{A} \stackrel{\mathrm{b}^{\prime}}{\rightarrow} \mathcal{A} \otimes \overline{\mathcal{A}} \otimes \mathcal{A} \stackrel{\mathrm{b}^{\prime}}{\rightarrow} \mathcal{A} \otimes \mathcal{A} \stackrel{\mathrm{b}^{\prime}}{\rightarrow} \mathcal{A} \rightarrow 0,
$$

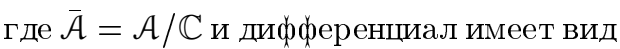

$$
\mathrm{b}^{\prime} a_{0} \otimes a_{1} \otimes \cdots \otimes a_{n}=\sum_{i=0}^{n-1}(-1)^{i} a_{0} \otimes \cdots \otimes a_{i-1} \otimes a_{i} a_{i+1} \otimes a_{i+2} \otimes \cdots \otimes a_{n} .
$$

Данный комплекс является стягиваемым, причем стягиваюшая гомотопия есть s:

$$
\mathrm{sb}^{\prime}+\mathrm{b}^{\prime} \mathrm{s}=\mathrm{id} .
$$

Для установления связи с содержанием раздела 2 определим линейные отображения $\mathrm{Z}^{n}: \mathcal{A}^{\otimes n} \rightarrow \mathcal{A}^{\otimes n}$ как

$$
\mathrm{Z}=\mathrm{b}^{\prime} \mathrm{s} \text {. }
$$

Их предстоит рассматривать как (квантовые) отображения $\stackrel{n}{Z}$ из раздела 2 . Явным образом имеем

$$
Z^{2} a \otimes b=a \otimes b-1 \otimes a b, \quad Z^{3} a \otimes b \otimes c=a \otimes b \otimes c-1 \otimes a b \otimes c+1 \otimes a \otimes b c
$$

и т.д. Для последуюшего использования введем также обозначение

$$
Z_{1, i}^{j}=\mathrm{P}_{1, i} \circ\left(\mathrm{id}^{\otimes(i-1)} \otimes \mathrm{Z}^{j}\right): \mathcal{A}^{\otimes(i+j-1)} \rightarrow \mathcal{A}^{\otimes(i+j-1)} .
$$

Очевидно, что $\mathrm{m} \circ \mathrm{Z}^{2}=0$; в действительности имеется точная последовательность $0 \rightarrow \mathcal{A} \otimes \overline{\mathcal{A}} \stackrel{\mathrm{z}^{2}}{\rightarrow} \mathcal{A} \otimes \mathcal{A} \stackrel{\mathrm{m}}{\rightarrow} \mathcal{A}$. Чтобы получить аналогичное утверждение об обращении в нуль для высших $Z^{n}$, составим композишию $Z^{n}$ и $Z^{n-1}$ путем “дополнения" младшего из двух тождественным отображением слева. 
ЛЕмма 3.1. При $n \geqslant 2$ имеем

$$
\mathrm{Z}^{n} \circ\left(\mathrm{id}_{1} \otimes \mathrm{Z}^{n-1}\right)=\left(\mathrm{id}_{1} \otimes \mathrm{Z}^{n-1}\right) \circ \mathrm{Z}^{n}=\left(\mathrm{Z}^{2} \otimes \mathrm{id}^{\otimes(n-2)}\right) \circ\left(\mathrm{id}_{1} \otimes \mathrm{Z}^{n-1}\right),
$$

поэтому сквозные отображсения

$$
\begin{aligned}
& \mathcal{A}^{\otimes n} \stackrel{\text { id } \otimes \mathbf{Z}^{n-1}}{\longrightarrow} \mathcal{A}^{\otimes n} \stackrel{\mathrm{Z}^{n}}{\longrightarrow} \mathcal{A}^{\otimes n} \stackrel{\mathrm{\textrm {m } _ { 1 2 }}}{\longrightarrow} \mathcal{A}^{\otimes(n-1)}, \\
& \mathcal{A}^{\otimes n} \stackrel{\mathrm{Z}^{n}}{\longrightarrow} \mathcal{A}^{\otimes n} \stackrel{\mathrm{id} \otimes \mathrm{Z}^{n-1}}{\longrightarrow} \mathcal{A}^{\otimes n} \stackrel{\mathrm{m}_{12}}{\longrightarrow} \mathcal{A}^{\otimes(n-1)}
\end{aligned}
$$

тождественно нулевъе.

Эта лемма объясняет интерпретацию $Z^{n}$ в духе калибровочной теории как “нулевых мод" $\stackrel{n}{Z}$ из раздела 2 . Пусть $t_{a}-$ базис в $\mathcal{A}$ и

$$
t_{a} t_{b}=\sum_{c} f_{a b}^{c} t_{c}
$$

- таблица умножения алгебры. Тогда аналог соотношений (1.1) очевидно имеет вид

$$
\left[t_{a}, t_{b}\right]=\sum_{c} f_{[a b]}^{c} t_{c}
$$

Далее, для элементов $a=\sum_{c} t_{c} a^{c}$ запишем

$$
Z^{n}\left(a_{1} \otimes \cdots \otimes a_{n}\right)=Z_{b_{1} \ldots b_{n}}^{c_{1} \ldots c_{n-1}} \otimes t_{c_{1}} \otimes \cdots \otimes t_{c_{n-1}} a_{1}^{b_{1}} \ldots a_{n}^{b_{n}}, \quad Z_{b_{1} \ldots b_{n}}^{c_{1} \ldots c_{n-1}} \in \mathcal{A}
$$

где подразумевается суммирование по повторяюшимся индексам. Первый тензорный множитель в образе $Z^{n}$ тем самым отделен от остальных: каждое $Z_{b_{1} \ldots b_{n}}^{c_{1} \ldots c_{n-1}}$ является элементом в этом экземпляре алгебры $\mathcal{A}$. В таком компонентном виде $Z^{2}$ представляется как $Z_{b c}^{a}=t_{b} \delta_{c}^{a}-f_{b c}^{a}$, а выписанное выше соотношение $\mathrm{m} \circ \mathrm{Z}^{2}=0$ принимает вид

$$
\sum_{c} Z_{a b}^{c} t_{c}=0
$$

эти равенства надлежит рассматривать как условия приводимости (2.1), Далее, соотношения приводимости для высших отображений $Z^{n}$ - аналоги (2.2) - являются условиями обрашения в нуль из леммы 3.1 , которые в компонентных обозначениях принимают вид

$$
\sum_{a_{1}, \ldots, a_{n}} Z_{b_{1} \ldots b_{n+1}}^{a_{1} \ldots a_{n}} Z_{a_{1} \ldots a_{n}}^{c_{1} \ldots c_{n-1}}=0
$$

(что включает умножение в $\mathcal{A}$ ). Более точно равенства $(3.7)$ интерпретируются как "квантовъе" уравнения (2.4) в том частном случае, когда $\Pi=0$ и в правой части не возникают члены $\hbar \mathbb{A}^{\hbar}$. 
3.2. Некоммутативные дифференциальные формы. Напомним интерпретацию $\Omega^{n}=\mathcal{A} \otimes \overline{\mathcal{A}}^{\otimes n}$ как некоммутативных дифференциальных форм [9], [10]. Алгебра некоммутативных дифференциальных форм $\Omega^{\bullet}=\Omega^{\bullet} \mathcal{A}$ над $\mathcal{A}$ есть универсальная дифференциальная градуированная алгебра, порожденная $\mathcal{A}$ и символами $d a, a \in \mathcal{A}$, причем $d a$ линейно по $a$, выполняется правило Лейбница $d(a b)=d(a) b+a d b$ и $d 1=0$. Изоморфизм $\mathcal{A} \otimes \overline{\mathcal{A}}^{\otimes n} \rightarrow \Omega^{n}$ есть $a_{0} \otimes a_{1} \otimes \cdots \otimes a_{n} \mapsto a_{0} d a_{1} \ldots d a_{n}$. При этом изоморфизме действие $d$ принимает вид $d\left(a_{0} \otimes a_{1} \otimes \cdots \otimes a_{n}\right)=1 \otimes a_{0} \otimes a_{1} \otimes \cdots \otimes a_{n}$.

Некоммутативные дифференциальные формы являются бимодулем над $\mathcal{A}$. Левое действие очевидно, а алгоритм определения правого действия таков: на 1-формах элемент $c \in \mathcal{A}$ действует как $a_{0} d a_{1} . c=a_{0} d\left(a_{1} c\right)-a_{0} a_{1} d c$, и аналогичным же образом на старших формах, начиная справа и "продвигаясь" налево с использованием правила Лейбница, пока все члены не примут вид $b_{0} d b_{1} \ldots d b_{n}$, например,

$$
a_{0} d a_{1} d a_{2} \cdot c=a_{0} d a_{1} d\left(a_{2} c\right)-a_{0} d\left(a_{1} a_{2}\right) d c+a_{0} a_{1} d a_{2} d c .
$$

Пусть $\mathrm{L}_{D}$ при $D \in \operatorname{Hom}(\mathcal{A}, \mathcal{A})$ обозначает производную Ли, действуюшую на некоммутативные дифференциальные формы как [10]

$$
\begin{aligned}
\mathrm{L}_{D}\left(a_{0} d a_{1} \ldots d a_{n}\right)= & D\left(a_{0}\right) d a_{1} \ldots d a_{n}+ \\
& +a_{0} d D\left(a_{1}\right) d a_{2} \ldots d a_{n}+\cdots+a_{0} d a_{1} \ldots d a_{n-1} d D\left(a_{n}\right),
\end{aligned}
$$

и пусть также $I_{D}$ обозначает свертку

$$
\begin{aligned}
\mathrm{I}_{D}\left(a_{0} d a_{1} \ldots d a_{n}\right)= & a_{0} D\left(a_{1}\right) d a_{2} \ldots d a_{n}- \\
& -a_{0} d a_{1} \cdot D\left(a_{2}\right) d a_{3} \ldots d a_{n}+\cdots+(-1)^{n-1} a_{0} d a_{1} \ldots d a_{n-1} \cdot D\left(a_{n}\right) .
\end{aligned}
$$

Тогда имеет место картановская формула гомотопии $\mathrm{L}_{D}=\mathrm{I}_{D} d+d \mathrm{I}_{D}$. Для $a \in \mathcal{A}$ и внутреннего дифференцирования $[a,-]$ мы, злоупотребляя обозначениями, будем писать $\mathrm{L}_{a}=\mathrm{L}_{[a,-]}$ и $\mathrm{I}_{a}=\mathrm{I}_{[a,-]}$.

Лемма 3.2. Если $D \in \operatorname{Hom}(\mathcal{A}, \mathcal{A})$ - дифференцирование, то $\mathrm{I}_{D} \mathrm{~b}^{\prime}+\left.\mathrm{b}^{\prime}\right|_{D}=0 u$ $\mathrm{b}^{\prime} \mathrm{L}_{D}-\mathrm{L}_{D} \mathrm{~b}^{\prime}=0$.

Первое соотношение проверяется непосредственно, а в комбинации с формулой гомотопии оно немедленно приводит ко второму соотношению. Данная лемма используется ниже для внутренних дифференцирований $D=[a,-]$; в частности, $\mathrm{b}^{\prime} \mathrm{L}_{a}=\mathrm{L}_{a} \mathrm{~b}^{\prime}$ при $a \in \mathcal{A}$.

Мы распространим приведенные вьше соотношения на bar-резольвенту $\Omega^{\bullet} \otimes \mathcal{A}$.

3.3. Духи и БРСТ-дифференциал $\boldsymbol{\Omega}$. Мы собираемся рассматривать bar-peзольвенту и отображения $Z^{n}$ как приводимую калибровочную теорию, в которой $t_{a}$ (выбранный базис в $\mathcal{A}$ ) играют роль связей, а соотношения приводимости даются формулами (3.6) и (3.7).

3.3.1. Состав духов. Введем духи для каждого члена bar-резольвенты: $C^{n} \in \Omega^{n-2} \otimes$ $\mathcal{A}, n \geqslant 2$, и $C_{1} \in \mathcal{A}$, а также сопряженные импульсы $P_{n} \in \operatorname{Hom}\left(\overline{\mathcal{A}}^{\otimes(n-1)} \otimes \mathcal{A}, \mathbb{C}\right), n \geqslant 2$, и $P_{1} \in \operatorname{Hom}(\mathcal{A}, \mathbb{C})$ с $\mathbb{Z}_{2}$-градуировками $\varepsilon\left(C^{n}\right)=\varepsilon\left(P_{n}\right) \equiv n \bmod 2$. Припишем духовые 
числа gh $C^{n}=n, \operatorname{gh} P_{n}=-n$. Тогда $\mathbb{Z}_{2}$-градуировка совпадает с градуировкой по духовому числу, рассматриваемой по модулю 2 ; тем не менее мы будем явно указывать $\mathbb{Z}_{2}$-градуировку наряду с духовым числом.

Если элементам $a_{i} \in \mathcal{A}$ приписаны $\mathbb{Z}_{2}$-градуировки $\varepsilon\left(a_{i}\right)$, мы полагаем $\varepsilon\left(a_{1} \otimes \cdots \otimes\right.$ $\left.a_{n}\right)=\varepsilon\left(a_{1}\right)+\cdots+\varepsilon\left(a_{n}\right)$ и иногда будем писать $\varepsilon(a)$ при $\varepsilon\left(a_{1} \otimes \cdots \otimes a_{n}\right)$.

Запишем каноническое спаривание как

$$
\begin{gathered}
\left(\overline{\mathcal{A}}^{\otimes n} \otimes \mathcal{A}\right) \otimes \operatorname{Hom}\left(\overline{\mathcal{A}}^{\otimes n} \otimes \mathcal{A}, \mathbb{C}\right) \rightarrow \mathbb{C}, \\
\left(a_{1} \otimes \cdots \otimes a_{n} \otimes a^{\prime}\right) \otimes D \mapsto\left\langle a_{1} \otimes \cdots \otimes a_{n} \otimes a^{\prime}, D\right\rangle .
\end{gathered}
$$

Оно индуцирует также свертку

$$
\begin{gathered}
\left(\mathcal{A} \otimes \overline{\mathcal{A}}^{\otimes n} \otimes \mathcal{A}\right) \otimes \operatorname{Hom}\left(\overline{\mathcal{A}}^{\otimes n} \otimes \mathcal{A}, \mathbb{C}\right) \rightarrow \mathcal{A}, \\
\left(a_{0} \otimes \cdots \otimes a_{n} \otimes a^{\prime}, D\right) \mapsto \iota\left(a_{0} \otimes \cdots \otimes a_{n} \otimes a^{\prime}\right)_{D},
\end{gathered}
$$

где $\iota\left(a_{0} \otimes \cdots \otimes a_{n} \otimes a^{\prime}\right)_{D}=a_{0}\left\langle a_{1} \otimes \cdots \otimes a_{n}, D\right\rangle$.

3.3.2. Дифференциальные операторы на bar-резольвенте и $\Omega$. Предписание гамильтонова квантования включает каноническое квантование духов [2]; в нашем случае это приводит к рассмотрению дифференциальных операторов на bar-резольвенте. Каждая пара $C^{n}, P_{n}$ удовлетворяет каноническим коммутационным соотношениям (мнемонически $\left[C^{n}, P_{m}\right]=\delta_{m}^{n}$, а потому $\left.\left[P_{n}, C^{m}\right]=(-1)^{n+1} \delta_{n}^{m}\right)$, и коммутирование $C^{n}$ через $P_{n}$, вычисленное на $a_{1} \otimes \cdots \otimes a_{n}$ в соответствии с $(3.8)$, дает

$$
C^{n}\left\langle a_{1} \otimes \cdots \otimes a_{n}, P_{n}\right\rangle=(-1)^{n \varepsilon(a)} a_{1} \otimes \cdots \otimes a_{n}+(-1)^{n(\varepsilon(a)+1)}\left\langle a_{1} \otimes \cdots \otimes a_{n}, P_{n}\right\rangle C^{n}
$$

или, другими словами,

$$
\left[C^{n},\left\langle a_{1} \otimes \cdots \otimes a_{n}, P_{n}\right\rangle\right]=(-1)^{n \varepsilon(a)} a_{1} \otimes \cdots \otimes a_{n} .
$$

Аналогичным образом вычисляем коммутаторы, включаюшие свертку (3.9), например,

$$
\left[C^{1}, \iota\left(a_{0} \otimes a_{1}\right)_{P_{1}}\right]=\left[C^{1}, a_{0}\right]\left\langle a_{1}, P_{1}\right\rangle+(-1)^{\varepsilon\left(a_{0}\right)+\varepsilon\left(a_{1}\right)} a_{0} a_{1},
$$

где первое слагаемое в правой части дается коммутатором, а второе - умножением в $\mathcal{A}$.

Опираясь на опыт работы с БРСТ-формализмом, будем искать дифференциал $\boldsymbol{\Omega}$ в виде

$$
\boldsymbol{\Omega}=\boldsymbol{\Omega}_{0}+\boldsymbol{\Omega}_{\mathcal{A}}, \quad \operatorname{gh} \boldsymbol{\Omega}=1
$$

где

$$
\boldsymbol{\Omega}_{\mathcal{A}}=C^{1}+\sum_{n \geqslant 1} \iota\left(\mathrm{Z}^{n+1} C^{n+1}\right)_{P_{n}}
$$

включает “граничные" члены, явно выписанные в (2.7) (хотя и относится к бесконечно-приводимому случаю $\ell=\infty$ ), а

$$
\begin{aligned}
\boldsymbol{\Omega}_{0}= & \sum_{j \geqslant i \geqslant 1} \sum_{i \geqslant 1}\left\langle\mathrm{U}_{i, j}^{i+j-1}\left(C^{i} \otimes C^{j}\right), P_{i+j-1}\right\rangle+ \\
& +\sum_{j \geqslant i \geqslant 1} \sum_{m=1}^{(i+j-2) / 2}\left\langle\mathrm{U}_{i, j}^{m, i+j-m-1}\left(C^{i} \otimes C^{j}\right), P_{m} \otimes P_{i+j-m-1}\right\rangle
\end{aligned}
$$


с подлежащими определению “коэффициентами” U есть БРСТ-дифференциал для приводимой калибровочной теории с замкнутой алгеброй (см. замечания ниже по поводу его специальных свойств). Запишем также $\boldsymbol{\Omega}_{\mathcal{A}}$ в компонентных обозначениях (см. $(3.4)-(3.6))$ :

$$
\boldsymbol{\Omega}_{\mathcal{A}}=t_{a}\left(C^{1}\right)^{a}+\mathrm{Z}_{a b}^{2 c} C^{2 a b} P_{1 c}+\mathrm{Z}_{a b c}^{3 d e} C^{3 a b c} P_{2 d e}+\cdots
$$

3.4. Сформулируем теперь основную теорему о сушествовании БРСТ-дифференциала.

ТЕОрема 3.1. Существуют отображения

$$
\begin{aligned}
& \mathrm{U}_{m, m^{\prime}}^{n, n^{\prime}} \in \operatorname{Hom}\left(\mathcal{A}^{\otimes m} \otimes \mathcal{A}^{\otimes m^{\prime}}, \mathcal{A}^{\otimes n} \otimes \mathcal{A}^{\otimes n^{\prime}}\right), \\
& m+m^{\prime}=n+n^{\prime}+1, \quad 1 \leqslant n<n^{\prime}, \quad 1 \leqslant m \leqslant m^{\prime}, \\
& \mathrm{U}_{m, m^{\prime}}^{m+m^{\prime}-1} \in \operatorname{Hom}\left(\mathcal{A}^{\otimes m} \otimes \mathcal{A}^{\otimes m^{\prime}}, \mathcal{A}^{\otimes\left(m+m^{\prime}-1\right)}\right), \quad 1 \leqslant m \leqslant m^{\prime},
\end{aligned}
$$

такие, что для оператора $\Omega(3.12)-(3.14)$ выполнено $\Omega \Omega=0$.

ЗАмечАниЕ 3.1. Отображения $\mathrm{U}_{m, m^{\prime}}^{n, n^{\prime}}$, индексы которых не удовлетворяют ограничениям, указанным в теореме 3.1 , можно считать равными нулю. Равенства $U_{m, m^{\prime}}^{n, n^{\prime}}=0$ при $n>n^{\prime}$ являются вопросом соглашения (альтернативой были бы градуированные условия симметрии по отношению к перестановке индексов), но условия $\mathrm{U}_{m, m^{\prime}}^{n, n}=0$ существенны: они означают, что никакой духовый импульс не входит в БРСТ-дифференциал квадратично. Другая договоренность состоит в том, что $\bigcup_{m, m^{\prime}}^{n, n^{\prime}}=0$ при $m>m^{\prime}$.

ЗАмЕчАниЕ 3.2 . Заметим, что оператор $\boldsymbol{\Omega}_{\mathcal{A}}$ в $(3.13)$ линеен как по духам, так и по импульсам, причем коэффициенты суть отображения $\mathrm{Z}^{n}(3.2)$, а потому $\boldsymbol{\Omega}_{\mathcal{A}}$ является дифференциальным оператором с коэффициентами в $\mathcal{A}$ (ср. текст после леммы 3.1$)$ и представляет собой не что иное, как “граничные члены” (ср. (2.7)). С другой стороны, оператор $\boldsymbol{\Omega}_{0}$ билинеен по духам и (отдельно) по импульсам, но является оператором со скалярными коэффициентами ввиду наличия в нем сверток $\langle-, P\rangle_{\text {и }}\langle-, P \otimes P\rangle$. Общая структура $\Omega$ та же, что описана в $(2.12)$, с тем дополнительным свойством, что $\tau_{A}$ линейны, а $U_{A B}$ максимум билинейны по духовым импульсам. Условия (2.13) выполнены в силу того, что $\tau_{A}$ и $U_{A B}$ являются функциями только импульсов, а $U_{A B}$, как было отмечено, - скаляры.

ЗАмЕчАниЕ 3.3. Для дифференшиала $\Omega$ из теоремы $3.1 \Omega_{0}$ не является дифференциалом.

3.5. Решение для $U_{m, m^{\prime}}^{n, n^{\prime}}$ и $U_{m, m^{\prime}}^{m+m^{\prime}-1}$ из теоремы 3.1. Найдем отображения $U$, решив часть уравнений, следуюших из $\Omega \Omega=0$, а затем покажем, что остаюшиеся уравнения также выполнены. Начнем с вычисления $\Omega \Omega$ для оператора (3.12)-(3.14).

3.5.1. Вычисление $\boldsymbol{\Omega}^{2}$. Простое вычисление с использованием (3.10), (3.11) показывает, что

$$
\boldsymbol{\Omega}_{\mathcal{A}} \boldsymbol{\Omega}_{\mathcal{A}}=C^{1} C^{1}+\sum_{m \geqslant 1} \iota\left(z_{m}\right)_{P_{m}}+\sum_{n \geqslant m \geqslant 1} \sum_{m \geqslant} \iota\left(z_{m n}\right)_{P_{m} \otimes P_{n}}
$$


где первый член в правой части дается умножением в алгебре, а члены ряда имеют вид $\iota(-)_{P}$-сверток от выражений

$$
\begin{aligned}
z_{m} & =\underset{1}{\operatorname{ad}} C^{1} \cdot \mathbf{Z}^{m+1}\left(C^{m+1}\right), \\
z_{n n} & =\zeta_{n}\left(C^{n+1} \otimes C^{n+1}\right), \\
z_{m n} & =(-1)^{m n+m}\left(\mathrm{~m}_{1, m+2}-\mathrm{m}_{m+2,1}^{*}\right)\left(\mathbf{Z}^{m+1} C^{m+1} \otimes \mathbf{Z}^{n+1} C^{n+1}\right), \quad m<n .
\end{aligned}
$$

Здесь

$\zeta_{n}\left(a_{1} \otimes \cdots \otimes a_{n+1} \otimes b_{1} \otimes \cdots \otimes b_{n+1}\right)=\frac{1}{4}\left(\left[a_{1}, b_{1}\right] \otimes a_{2} \otimes \cdots \otimes a_{n+1} \otimes b_{2} \otimes \cdots \otimes b_{n+1}-\right.$ $\left.-(-1)^{n}\left[a_{1}, b_{1}\right] \otimes b_{2} \otimes \cdots \otimes b_{n+1} \otimes a_{2} \otimes \cdots \otimes a_{n+1}\right)$.

Используя далее (3.10), (3.11), получаем

$$
\left[\boldsymbol{\Omega}_{0}, \boldsymbol{\Omega}_{\mathcal{A}}\right]=\mathrm{U}_{1,1}^{1}\left(C^{1} \otimes C^{1}\right)+\sum_{m \geqslant 1} \iota\left(y_{m}\right)_{P_{m}}+\sum_{n \geqslant m \geqslant 1} \sum_{m} \iota\left(y_{m n}\right)_{P_{m} \otimes P_{n}},
$$

где члены ряда даются $\iota(-)_{P}$-свертками от выражений

$$
\begin{aligned}
& y_{m n}=(-1)^{n} Z_{1, m+1}^{n+1} \circ \sum_{\substack{j \geqslant i \geqslant 0 \\
i+j=m+n}} \mathrm{U}_{i+1, j+1}^{m, n+1}\left(C^{i+1} \otimes C^{j+1}\right)+ \\
& +\sum_{\substack{j>i \geqslant 0 \\
i+j=m+n}}(-1)^{m+n}\left(\mathrm{id} \otimes \mathrm{U}_{i+1, j}^{m, n}\right) \circ \mathbf{Z}_{1, i+2}^{j+1}\left(C^{i+1} \otimes C^{j+1}\right)+ \\
& +\sum_{\substack{j+1 \geqslant i \geqslant 1 \\
i+j=m+n}}(-1)^{i+1}\left(\mathrm{id} \otimes \mathrm{U}_{i, j+1}^{m, n}\right)\left(\mathrm{Z}^{i+1} C^{i+1} \otimes C^{j+1}\right)+ \\
& +(-1)^{m+n}\left(\mathrm{Z}^{m+1} \otimes \mathrm{id}^{\otimes n}\right) \circ \sum_{\substack{j \geqslant i \geqslant 0 \\
i+j=m+n}} \mathrm{U}_{i+1, j+1}^{m+1, n}\left(C^{i+1} \otimes C^{j+1}\right), \quad m<n, \\
& y_{n n}=(-1)^{n} \underset{2, n+1 ; n+2,2 n+1}{\mathrm{~S}^{(n)}}\left({\underset{1}{1, n+1}}^{n+1} \circ \sum_{i=0}^{n} \mathrm{U}_{i+1,2 n-i+1}^{n, n+1}\left(C^{i+1} \otimes C^{2 n-i+1}\right)\right) .
\end{aligned}
$$

Мы пользуемся обозначениями (3.3), а оператор $\underset{p, q ; r, s}{\mathrm{~S}_{(1)}^{(n)}}$, где $n$ рассматривается по модулю 2 и $p \leqslant q<r \leqslant s$ таковы, что $s-r=q-p$, осуществляет градуированную симметризацию (при четных $n$ ) или антисимметризацию (при нечетных $n$ ) тензорных множителей в позициях $[p, \ldots, q]$ и $[r, \ldots, s]$, например,

$\underset{2,3 ; 4,5}{\mathrm{~S}^{(n)}}(a \otimes b \otimes c \otimes d \otimes e)=\frac{1}{2} a \otimes b \otimes c \otimes d \otimes e+(-1)^{n} \cdot(-1)^{(\varepsilon(b)+\varepsilon(c))(\varepsilon(d)+\varepsilon(e))} \frac{1}{2} a \otimes d \otimes e \otimes b \otimes c$.

Кроме того, оказывается, что $y_{m}=y_{0, m}$, если положить

$$
\mathrm{U}_{i, j}^{0, \ell}=\mathrm{U}_{i, j}^{\ell} .
$$


В итоге вычисление $\boldsymbol{\Omega}_{0} \boldsymbol{\Omega}_{0}$ дает

$$
\Omega_{0} \Omega_{0}=\sum_{m \geqslant 2}\left\langle X_{m}, P_{m}\right\rangle+\sum_{\substack{m \geqslant 1 \\ m \leqslant n}} \sum_{\substack{n \geqslant 1 \\ m n}}\left\langle X_{m}, P_{m} \otimes P_{n}\right\rangle+X^{(3)},
$$

где

$$
\begin{aligned}
X_{m n}= & \sum_{\substack{i \geqslant 1 \\
2 i \leqslant m+n+1 \\
2 j \leqslant m+n+2}}(-1)^{(i+1)(m+n)+1} \mathrm{U}_{i, m+n+1-i}^{m, n} \circ \mathrm{U}_{j, m+n+2-j}^{i, m+n+1-i}\left(C^{j} \otimes C^{m+n+2-j}\right), \\
&
\end{aligned}
$$

$X_{m}=X_{0 m}$ и $X^{(3)}$ обозначает члены третьего порядка по духам $C^{n}$. Квадрат оператора $\boldsymbol{\Omega}$ поэтому равен

$\boldsymbol{\Omega} \boldsymbol{\Omega}=z_{0}+\sum_{m \geqslant 1} \iota\left(z_{m}+y_{m}+1 \otimes X_{m}\right)_{P_{m}}+\sum_{n \geqslant m \geqslant 1} \sum_{m \geqslant} \iota\left(z_{m n}+y_{m n}+1 \otimes X_{m n}\right)_{P_{m} \otimes P_{n}}+X^{(3)}$,

где $z_{0}=C^{1} C^{1}+\mathrm{U}_{1,1}^{1}\left(C^{1} \otimes C^{1}\right)$ и $z_{m}, y_{m}, x_{m}, z_{m n}, y_{m n}, x_{m n}$ при $n \geqslant m \geqslant 1$ приведены выше (как мы видели, $X_{1}=0$, а также $X_{n n}=0$ ). Разлагая (3.16) по духам и импульсам и приравнивая каждую степень нулю, получаем конкретную форму уравнений (2.14)-(2.16). В частности, кубичные по $C^{n}$ уравнения $X^{(3)}=0$ есть не что иное, как перезапись (2.16). Остальные члены в (3.16) не более чем квадратичны по духам $C^{n}$. Их зависимость от ду ховых импульсов указана в (3.16) явно, и мы поэтому сначала разложим $\boldsymbol{\Omega} \boldsymbol{\Omega}$ по импульсам. Уравнения $\boldsymbol{\Omega} \boldsymbol{\Omega}=0$ эквивалентны, таким образом, системе уравнений, состоящей из $\chi^{(3)}=0$ и

$$
\begin{aligned}
& z_{m}+y_{m}+1 \otimes x_{m}=0, \quad 1 \leqslant m, \\
& z_{m n}+y_{m n}+1 \otimes x_{m n}=0, \quad 1 \leqslant m<n, \\
& \underset{2, n+1 ; n+2,2 n+1}{\mathrm{~S}^{(n)}}\left(Z_{n n}+y_{n n}+1 \otimes X_{n n}\right)=0, \quad 1 \leqslant n .
\end{aligned}
$$

Дальнейшее разложение этих уравнений по духам $C^{n}$ дает бесконечный набор уравнений, начинающийся с (2.8)-(2.11). Говоря об уравнениях $z_{m n}+y_{m n}+1 \otimes x_{m n}=0$

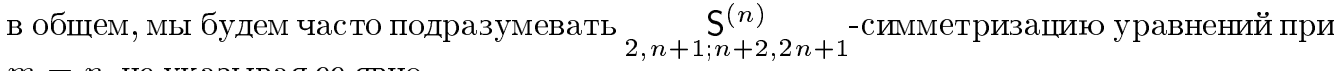
$m=n$, не указывая ее явно.

Мы собираемся решить уравнения (3.17), взяв некоторую их проекцию, которая даст набор рекурсионных соотношений для искомых отображений U.

3.5.2. Нахождение младших отображений. Члены низшего порядка в (3.16) $z_{0}$ обращаются в нуль, если $\mathrm{U}_{1,1}^{1}=-\mathrm{m}$. Поскольку $C^{1}$ является $\mathbb{Z}_{2}$-нечетным, вклад дает только антисимметричная часть умножения, и мы имеем

$$
\mathrm{U}_{1,1}^{1}=-\frac{1}{2}[-,-]
$$

В порядке $P_{1}$ легко находим, что $z_{1}+y_{1}=\mathrm{L}_{C^{1}} \cdot \mathrm{Z}^{2} C^{2}-\mathrm{Z}^{2} \circ \mathrm{U}_{1,2}^{2}\left(C^{1} \otimes C^{2}\right)$. Однако в силу леммы 3.2

$$
\mathrm{L}_{a} \circ \mathrm{Z}^{2}=\mathrm{L}_{a} \mathrm{~b}^{\prime} d=\mathrm{b}^{\prime} \mathrm{L}_{a} d=\mathrm{b}^{\prime}\left(d \mathrm{l}_{a}\right) d=\mathrm{b}^{\prime} d\left(\mathrm{I}_{a} d+d \mathrm{l}_{a}\right)=\mathrm{Z}^{2} \circ \mathrm{L}_{a},
$$


откуда видно, что $z_{1}+y_{1}=0$, если

$$
\mathrm{U}_{1,2}^{2}\left(C^{1} \otimes C^{2}\right)=\mathrm{L}_{C^{1}} C^{2} .
$$

Заметим, что тем самым решено уравнение (2.8) в наборе (2.8)-(2.11).

3.5.3. Установление рекурсии. Запишем отображения $\mathrm{Z}^{n}$ как $\mathrm{Z}=\mathrm{id}-1 \otimes \mathrm{b}^{\prime}$ и в каждом уравнении (3.17) рассмотрим члены, не имеющие вида $1 \otimes \cdots$. Операция $\mathcal{P}_{\not \chi}$ проектирования на такие члены сводится к отбрасыванию явных появлений $1 \otimes \cdots$ (в частности, $\left.1 \otimes X_{m n}\right)$ и к заменам $Z^{n} \rightarrow \mathrm{id}^{\otimes n}$ и $Z_{1, i}^{n} \rightarrow \mathrm{P}_{1, i}$. Из уравнений $(3.17)$ таким образом можно получить уравнения

$$
\begin{aligned}
& \sum_{i=0}^{[(m+n) / 2]} \mathrm{U}_{i+1, m+n-i+1}^{m, n+1}\left(C^{i+1} \otimes C^{m+n-i+1}\right)= \\
& =-(-1)^{m n+m+n} \mathrm{P}_{m+1,1} \circ\left(\mathrm{m}_{1, m+2}-\mathrm{m}_{m+2,1}^{*}\right)\left(C^{m+1} \otimes C^{n+1}\right)- \\
& \quad-(-1)^{m} \sum_{i=0}^{[(m+n-1) / 2]} \mathrm{P}_{m+1,1} \circ\left(\mathrm{id} \otimes \mathrm{U}_{i+1, m+n-i}^{m, n}\right) \circ \mathrm{P}_{1, i+2}\left(C^{i+1} \otimes C^{m+n-i+1}\right)+ \\
& \quad+\sum_{i=1}^{[(m+n+1) / 2]}(-1)^{i+n} \mathrm{P}_{m+1,1} \circ\left(\mathrm{id} \otimes \mathrm{U}_{i, m+n-i+1}^{m, n}\right)\left(C^{i+1} \otimes C^{m+n-i+1}\right)- \\
& \quad-(-1)^{m} \sum_{i=0}^{[(m+n) / 2]} \mathrm{P}_{m+1,1} \circ \mathrm{U}_{i+1, m+n-i+1}^{m+1, n}\left(C^{i+1} \otimes C^{m+n-i+1}\right),
\end{aligned}
$$

где $0 \leqslant m \leqslant n$ (при $m=0$ напомним равенства (3.15)). Каждое из этих соотношений сводится к $([(m+n) / 2]+1-m)$ независимым уравнениям для каждой пары духов $\left(C^{a}, C^{b}\right)$, но для экономии места мы будем держать их в виде "производящих соотношений”. Заметим, что при применении отображений к $C^{\ell} \otimes C^{\ell}$ следует выполнить градуированную симметризацию по отношению к двум "половинам" тензорного аргумента в соответствии с градуировкой $\varepsilon\left(C^{\ell}\right)=\ell$.

Чтобы увидеть, что равенства $(\boldsymbol{m}, \boldsymbol{n})$ являются в действительности набором рекурсионных соотношений, фиксируем $m+n=N$ при положительном целом $N$ и упорядочим $([N / 2]+1)([N / 2]+2) / 2$ уравнений, следуюших из $(\boldsymbol{m}, \boldsymbol{n})$, в порядке, определямом как

$$
\begin{aligned}
& \left.(m, n)=\left(\left[\frac{N}{2}\right],\left[\frac{N+1}{2}\right]\right) \quad \text { (1 уравнение }\right), \\
& (m, n)=\left(\left[\frac{N}{2}\right]-1,\left[\frac{N+1}{2}\right]+1\right) \quad(2 \text { уравнения }), \\
& (m, n)=(1, N-1) \quad\left(\left[\frac{N}{2}\right] \text { уравнений }\right), \\
& (m, n)=(0, N) \quad\left(\left[\frac{N}{2}\right]+1 \text { уравнений }\right) .
\end{aligned}
$$

Соответствуюший частичный порядок определяется при этом как

$$
\mathrm{U}_{m, n}^{m^{\prime}, n^{\prime}} \prec \mathrm{U}_{m_{1}, n_{1}}^{m_{1}^{\prime}, n_{1}^{\prime}},
$$


если $m^{\prime}+n^{\prime}<m_{1}^{\prime}+n_{1}^{\prime}$ или $m^{\prime}+n^{\prime}=m_{1}^{\prime}+n_{1}^{\prime}, n^{\prime}-m^{\prime}<n_{1}^{\prime}-m_{1}^{\prime}$. Можно видеть, что для каждого целого $N \geqslant 4$ обращающиеся в нуль отображения $\mathrm{U}_{m, n}^{m^{\prime}, n^{\prime}}$ суть

$$
\mathrm{U}_{m, n}^{m^{\prime}, m+n-m^{\prime}-1}=0, \quad m^{\prime} \geqslant m, \quad n-m \geqslant 2 .
$$

Рекурсионные соотношения удобно записать при нечетных и четных $m+n$ по отдельности.

3.5.4. Нечетные $(m+n)$. При $m+n=2 k-1$ уравнения $(\boldsymbol{m}, \boldsymbol{n})$, которые можно теперь обозначить как $(2 \boldsymbol{k}-1, \boldsymbol{m})$, где $0 \leqslant m \leqslant k-1$, принимают вид

$$
\begin{aligned}
& \sum_{i=m}^{k-1} \mathrm{U}_{i+1,2 k-i}^{m, 2 k-m}\left(C^{i+1} \otimes C^{2 k-i}\right)=\mathrm{P}_{m+1,1} \circ\left(\left(\mathrm{m}_{1, m+2}-\mathrm{m}_{m+2,1}^{*}\right)\left(C^{m+1} \otimes C^{2 k-m}\right)-\right. \\
& \quad-(-1)^{m} \sum_{i=m}^{k-1}\left(\mathrm{id} \otimes \mathrm{U}_{i+1,2 k-i-1}^{m, 2 k-m-1}\right) \circ \mathrm{P}_{1, i+2}\left(C^{i+1} \otimes C^{2 k-i}\right)- \\
& \quad-\sum_{i=m+1}^{k}(-1)^{i+m}\left(\mathrm{id} \otimes \mathrm{U}_{i, 2 k-i}^{m, 2 k-m-1}\right)\left(C^{i+1} \otimes C^{2 k-i}\right)- \\
& \left.\quad-(-1)^{m} \sum_{i=m+1}^{k-1} \mathrm{U}_{i+1,2 k-i}^{m+1,2 k-m-1}\left(C^{i+1} \otimes C^{2 k-i}\right)\right) .
\end{aligned}
$$

Очевидно, что эти соотношения позволяют выразить $\mathrm{U}_{i+1,2 k-i}^{m, 2 k-m}$ через $\mathrm{U}_{a, b}^{c, d} \prec \mathrm{U}_{i+1,2 k-i}^{m, 2 k-m}$, а именно, в соответствии с частичным порядком сначала выберем старшее значение $m=$ $k-1$. Тогда последней суммы в правой части нет, и поэтому $\mathrm{U}_{k, k+1}^{k-1, k+1}$ выражается через $\mathrm{U}_{m, n}^{m^{\prime}, n^{\prime}}$ с $m^{\prime}+n^{\prime}=2 k-1<2 k$, т.e.

$$
\begin{aligned}
& \mathrm{U}_{k, k+1}^{k-1, k+1}\left(C^{k} \otimes C^{k+1}\right)=\mathrm{P}_{k, 1} \circ\left(\left(\mathrm{m}_{1, k+1}-\mathrm{m}_{k+1,1}^{*}\right)\left(C^{k} \otimes C^{k+1}\right)+\right. \\
& \quad+(-1)^{k}\left(\mathrm{id} \otimes \mathrm{U}_{k, k}^{k-1, k}\right) \circ \mathrm{P}_{1, k+1}\left(C^{k} \otimes C^{k+1}\right)+ \\
& \left.\quad+\left(\mathrm{id} \otimes \mathrm{U}_{k, k}^{k-1, k}\right)\left(C^{k+1} \otimes C^{k}\right)\right) .
\end{aligned}
$$

Полагая далее $m=k-2$, получаем два уравнения

$$
\begin{gathered}
\mathrm{U}_{k-1, k+2}^{k-2, k+2}=\mathrm{P}_{k-1,1} \circ\left(\mathrm{m}_{2,1}^{*}-\mathrm{m}_{1,2}-(-1)^{k}\left(\mathrm{id} \otimes \mathrm{U}_{k-1, k+1}^{k-2, k+1}\right)\right) \circ \mathrm{P}_{1, k}, \\
\mathrm{U}_{k, k+1}^{k-2, k+2}\left(C^{k} \otimes C^{k+1}\right)=\mathrm{P}_{k-1,1} \circ\left(-(-1)^{k} \mathrm{U}_{k, k+1}^{k-1, k+1}\left(C^{k} \otimes C^{k+1}\right)+\right. \\
\quad+\left(\mathrm{id} \otimes \mathrm{U}_{k-1, k+1}^{k-2, k+1}\right)\left(C^{k} \otimes C^{k+1}\right)-\left(\mathrm{id} \otimes \mathrm{U}_{k, k}^{k-2, k+1}\right)\left(C^{k+1} \otimes C^{k}\right)- \\
\left.\quad-(-1)^{k}\left(\mathrm{id} \otimes \mathrm{U}_{k, k}^{k-2, k+1}\right) \circ \mathrm{P}_{1, k+1}\left(C^{k} \otimes C^{k+1}\right)\right)
\end{gathered}
$$

и т.д. При каждом $0 \leqslant m \leqslant k-2$ уравнения для "обших" значений $i$, т.е. $m+1 \leqslant i \leqslant k-$ 1 , суть

$$
\begin{aligned}
\mathrm{U}_{i+1,2 k-i}^{m, 2 k-m}= & \mathrm{P}_{m+1,1} \circ\left((-1)^{m+1}\left(\mathrm{id} \otimes \mathrm{U}_{i+1,2 k-i-1}^{m, 2 k-m-1}\right) \circ \mathrm{P}_{1, i+2}-\right. \\
& \left.-\mathrm{id} \otimes \mathrm{U}_{i, 2 k-i}^{m, 2 k-m-1}-(-1)^{m} \mathrm{U}_{i+1,2 k-i}^{m+1,2 k-m-1}\right) .
\end{aligned}
$$

"Граничные" уравнения при $i=m$ и $i=k$ также легко получить из $(2 \boldsymbol{k}-1, \boldsymbol{m})$ (уравнение при $i=m$ включает "неоднородный" вклад $\mathrm{P}_{m+1,1} \circ\left(\mathrm{m}_{1, m+2}-\mathrm{m}_{m+2,1}^{*}\right)$ в правой части). 
3.5.5. Четные $(m+n)$. При $m+n=2 k$ уравнения $(\boldsymbol{m}, \boldsymbol{n})$, которые можно теперь обозначить как $(2 \boldsymbol{k}, \boldsymbol{m})$, где $0 \leqslant m \leqslant k$, принимают вид

$$
\begin{aligned}
& \sum_{i=m}^{k} \mathrm{U}_{i+1,2 k-i+1}^{m, 2 k-m+1}\left(C^{i+1} \otimes C^{2 k-i+1}\right)= \\
& =-(-1)^{m} \mathrm{P}_{m+1,1} \circ\left(\left(\mathrm{m}_{1, m+2}-\mathrm{m}_{m+2,1}^{*}\right)\left(C^{m+1} \otimes C^{2 k-m+1}\right)-\right. \\
& \quad-(-1)^{m} \sum_{i=m}^{k-1}\left(\mathrm{id} \otimes \mathrm{U}_{i+1,2 k-i}^{m, 2 k-m}\right) \circ \mathrm{P}_{1, i+2}\left(C^{i+1} \otimes C^{2 k-i+1}\right)+ \\
& \quad+\sum_{i=m+1}^{k}(-1)^{i+m}\left(\mathrm{id} \otimes \mathrm{U}_{i, 2 k-i+1}^{m, 2 k-m}\right)\left(C^{i+1} \otimes C^{2 k-i+1}\right)- \\
& \left.\quad-(-1)^{m} \sum_{i=m+1}^{k} \mathrm{U}_{i+1,2 k-i+1}^{m+1,2 k-m}\left(C^{i+1} \otimes C^{2 k-i+1}\right)\right)
\end{aligned}
$$

Их также следует рассматривать в порядке, определенном последовательностью значений $m=k, k-1, \ldots, 1$. Старшее значение $m=k$ здесь несколько специальное: с одной стороны, все отображения $U_{m, n}^{m^{\prime}, n^{\prime}}$ выпадают из правой части и остается только первое слагаемое, а с другой стороны, выполнение уравнения требуется только после $\underset{\mathrm{S}}{\mathrm{S}}(k)$-симметризации (см. замечание после (3.17)) и с соответствующей сим$2, k+1 ; k+2,2 k+1$

метризацией ду хового аргумента $C^{k+1} \otimes C^{k+1}($ см. замечание после $(\boldsymbol{m}, \boldsymbol{n}))$. Это оставляет некоторую свободу в определении $\mathrm{U}_{k+1, k+1}^{k, k+1}$, которая фиксируется с использованием полного уравнения $\underset{2, k+1 ; k+2,2 k+1}{\mathrm{~S}}(k) \quad\left(\mathcal{Z}_{k k}+\mathcal{Y}_{k k}+1 \otimes X_{k k}\right)=0$ для $\mathrm{U}_{k, k}^{k-1, k}$. Тогда имеем

$$
\mathrm{U}_{k, k}^{k-1, k}=(-1)^{k+1} \mathrm{~b}^{\prime} \otimes \mathrm{id}^{\otimes k}+(-1)^{k}\left(\mathrm{id}{ }^{\otimes(k-1)} \otimes \mathrm{b}^{\prime}\right) \circ \mathrm{P}_{k, 1},
$$

где $\mathrm{P}_{k, 1}$ обозначает обратный к $\mathrm{P}_{1, k}$ оператор, и при вычислении $\mathrm{U}_{k, k}^{k-1, k}$ на $C^{k} \otimes C^{k}$ следует выполнить градуированную симметризацию по отношению к двум "половинам" тензорного аргумента, например (опуская знаковые множители, соответствующие

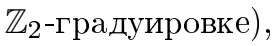

$$
\begin{aligned}
\mathrm{U}_{2,2}^{1,2} a \otimes b \otimes a^{\prime} \otimes b^{\prime}= & \frac{1}{2}\left(-a b \otimes a^{\prime} \otimes b^{\prime}+b \otimes a a^{\prime} \otimes b^{\prime}-b \otimes a \otimes a^{\prime} b^{\prime}-\right. \\
& \left.-a^{\prime} b^{\prime} \otimes a \otimes b+b^{\prime} \otimes a^{\prime} a \otimes b-b^{\prime} \otimes a^{\prime} \otimes a b\right)
\end{aligned}
$$

(что дает решение уравнения (2.10)).

Следующие (два) уравнения, вытекающие из $(2 \boldsymbol{k}, \boldsymbol{m})$, соответствуют $m=k-1$ и позволяют выразить $\mathrm{U}_{k, k+2}^{k-1, k+2}$ через $\mathrm{U}_{k, k+1}^{k-1, k+1}$ и $\mathrm{U}_{k+1, k+1}^{k-1, k+2}$ через $\mathrm{U}_{k, k+1}^{k-1, k+1}$ и $\mathrm{U}_{k+1, k+1}^{k, k+1}$; все эти уравнения легко записываются аналогично (3.26). Применяя рекурсию далее, следует только учесть, что при $0 \leqslant m \leqslant k-1$ уравнения

$$
\begin{aligned}
\mathrm{U}_{k+1, k+1}^{m, 2 k-m+1}\left(C^{k+1} \otimes C^{k+1}\right)= & (-1)^{k+m} \mathrm{P}_{m+1,1} \circ\left(\mathrm{id} \otimes \mathrm{U}_{k, k+1}^{m, 2 k-m}\right)\left(C^{k+1} \otimes C^{k+1}\right)- \\
& -(-1)^{m} \mathrm{P}_{m+1,1} \circ \mathrm{U}_{k+1, k+1}^{m+1,2 k-m}\left(C^{k+1} \otimes C^{k+1}\right),
\end{aligned}
$$


следующие из $(2 \boldsymbol{k}, \boldsymbol{m})$, вычисляются на $C^{k+1} \otimes C^{k+1}$, а потому отображения в правой части следует соответствуюшим образом симметризовать по отношению к двум "половинам" тензорного аргумента. Равенства $(3.18),(3.19),(3.22),(2 \boldsymbol{k}-1, \boldsymbol{m})$ и $(2 \boldsymbol{k}, \boldsymbol{m})$ определяют все отображения, входящие в дифференциал $\boldsymbol{\Omega}$.

Заметим еще, что структура, входящая в (3.19), проходит через рекурсионные соотношения как

$$
\mathrm{U}_{1, n}^{n} a_{0} \otimes a_{1} \otimes \cdots \otimes a_{n}=(-1)^{n} \mathrm{~L}_{a_{0}} a_{1} \otimes \cdots \otimes a_{n} .
$$

3.5.6. ПримеР. В дополнение к (3.18), (3.19), (3.28) (и (3.30)) приведем явную формулу, следуюшую из рекурсионных соотношений. Для $\mathrm{U}_{m, n}^{m^{\prime}, n^{\prime}}$ при $m^{\prime}+n^{\prime}=3$ наряду с (3.28) имеем

$$
\begin{aligned}
2 \mathrm{U}_{2,2}^{3} a & \otimes b \otimes a^{\prime} \otimes b^{\prime}=a \otimes a^{\prime} \otimes b^{\prime} b-a \otimes a^{\prime} \otimes b b^{\prime}+a \otimes a^{\prime} b \otimes b^{\prime}-a \otimes b a^{\prime} \otimes b^{\prime}+ \\
& +a^{\prime} \otimes a \otimes b b^{\prime}-a^{\prime} \otimes a \otimes b^{\prime} b+a^{\prime} \otimes a b^{\prime} \otimes b-a^{\prime} \otimes b^{\prime} a \otimes b+b \otimes a \otimes a^{\prime} b^{\prime}- \\
& -b \otimes a a^{\prime} \otimes b^{\prime}+b^{\prime} \otimes a^{\prime} \otimes a b-b^{\prime} \otimes a^{\prime} a \otimes b+a b \otimes a^{\prime} \otimes b^{\prime}+a^{\prime} b^{\prime} \otimes a \otimes b .
\end{aligned}
$$

3.6. Окончание доказательства уравнений (3.17). Мы определили рекуррентно найденные отображения $\mathrm{U}_{m, n}^{\ell, m+n-\ell-1}$ из $\mathcal{P}_{\chi}$-проекций уравнений (3.17). Теперь надо показать, что остаюшаяся часть уравнений, $z_{m n}+y_{m n}+1 \otimes X_{m n}=0,0 \leqslant m<n$, также выполнена. В явном виде уравнения, которые следует проверить, получаются заменой $Z \rightarrow-1 \otimes \mathrm{b}^{\prime}$; все тензорные члены тогда приобретают вид $1 \otimes \mathbb{X}$, и возникаюшие уравнения $\mathbb{X}=0$ (как и ранее, записанные как "производяшие") имеют вид

$$
\begin{aligned}
(-1)^{m}\left(\mathrm{id}^{\otimes m} \otimes \mathrm{b}^{\prime}\right) \circ \sum_{i=m}^{[(m+n) / 2]} \mathrm{U}_{i+1, m+n-i+1}^{m, n+1}\left(C^{i+1} \otimes C^{m+n-i+1}\right)+ \\
+\left(\mathrm{b}^{\prime} \otimes \mathrm{id} \mathrm{d}^{\otimes n}\right) \circ \sum_{i=m+1}^{[(m+n) / 2]} \mathrm{U}_{i+1, m+n-i+1}^{m+1, n}\left(C^{i+1} \otimes C^{m+n-i+1}\right)+ \\
+\sum_{i=m}^{[(m+n-1) / 2]} \mathrm{U}_{i+1, m+n-i}^{m, n}\left(C^{i+1} \otimes \mathrm{b}^{\prime} C^{m+n-i+1}\right)+ \\
+\sum_{i=m+1}^{[(m+n+1) / 2]}(-1)^{m+n+i+1} \mathrm{U}_{i, m+n-i+1}^{m, n}\left(\mathrm{~b}^{\prime} C^{i+1} \otimes C^{m+n-i+1}\right)+ \\
+\sum_{j=m+1} \sum_{i=j}^{[(m+n) / 2][(m+n) / 2]}(-1)^{j(m+n)} \times \\
\quad \times \mathrm{U}_{j, m+n+1-j}^{m, n} \circ \mathrm{U}_{i+1, m+n+1-i}^{j, m+n+1-j}\left(C^{i+1} \otimes C^{m+n+1-i}\right)=0
\end{aligned}
$$

при $0 \leqslant m<n$. Требуется показать, что они выполнены при отображениях $\mathrm{U}_{m, n}^{\ell, m+n-\ell-1}$, определенных установленной выше рекурсией.

3.6.1. Метод. Уравнения (3.31) доказываются (несколько утомительной) индукцией по порядку отображений $\mathrm{U}$, введенному в (3.21). Рассмотрим уравнения (3.31) при 
$m+n=2 k-1$ и предположим, что все уравнения при $m+n \leqslant 2 k-2$ выполнены (уравнения (3.31) при $m+n=2 k$ доказываются аналогично). Равенства, подлежашие проверке при $m+n=2 k-1$, таковы. Полагая $m=k-1-\ell$ и $n=k+\ell$, имеем $\ell+1$ уравнений для каждого $\ell \in\{0, \ldots, k-1\} ;$ при $\ell \geqslant 1$ это

$$
\begin{aligned}
& (-1)^{k+\ell}\left(\mathrm{id}^{\otimes(k-\ell-1)} \otimes \mathrm{b}^{\prime}\right) \circ \mathrm{U}_{k, k+1}^{k-\ell-1, k+\ell+1}\left(C^{k} \otimes C^{k+1}\right)- \\
& -\left(\mathrm{b}^{\prime} \otimes \mathrm{id}^{\otimes(k+\ell)}\right) \circ \mathrm{U}_{k, k+1}^{k-\ell, k+\ell}\left(C^{k} \otimes C^{k+1}\right)-\mathrm{U}_{k, k}^{k-\ell-1, k+\ell}\left(C^{k} \otimes \mathrm{b}^{\prime} C^{k+1}\right)+ \\
& +(-1)^{k} \mathrm{U}_{k-1, k+1}^{k-\ell-1, k+\ell}\left(\mathrm{b}^{\prime} C^{k} \otimes C^{k+1}\right)-(-1)^{k} \mathrm{U}_{k, k}^{k-\ell-1, k+\ell}\left(\mathrm{b}^{\prime} C^{k+1} \otimes C^{k}\right)- \\
& -\sum_{j=0}^{\ell-1}(-1)^{k+1+j} \mathrm{U}_{k-j-1, k+j+1}^{k-\ell-1, k+\ell} \circ \mathrm{U}_{k, k+1}^{k-j-1, k+j+1}\left(C^{k} \otimes C^{k+1}\right)=0, \\
& (-1)^{k+\ell}\left(\mathrm{id}^{\otimes(k-\ell-1)} \otimes \mathrm{b}^{\prime}\right) \circ \mathrm{U}_{k-\ell, k+\ell+1}^{k-\ell-1, k+\ell+1}-\mathrm{U}_{k-\ell, k+\ell}^{k-\ell-1, k+\ell} \circ\left(\mathrm{id}^{\otimes(k-\ell)} \otimes \mathrm{b}^{\prime}\right)=0, \\
& (-1)^{k+\ell}\left(\mathrm{id}^{\otimes(k-\ell-1)} \otimes \mathrm{b}^{\prime}\right) \circ \mathrm{U}_{k-i, k+i+1}^{k-\ell-1, k+\ell+1}-\left(\mathrm{b}^{\prime} \otimes \mathrm{id}^{\otimes(k+\ell)}\right) \circ \mathrm{U}_{k-i, k+i+1}^{k-\ell, k+\ell}- \\
& -\mathrm{U}_{k-i, k+i}^{k-\ell-1, k+\ell} \circ\left(\mathrm{id}^{\otimes(k-i)} \otimes \mathrm{b}^{\prime}\right)+(-1)^{k+i} \mathrm{U}_{k-i-1, k+i+1}^{k-\ell-1, k+\ell} \circ\left(\mathrm{b}^{\prime} \otimes \mathrm{id}^{\otimes(k+1+i)}\right)- \\
& -\sum_{j=i}^{\ell-1}(-1)^{k+1+j} \mathrm{U}_{k-j-1, k+j+1}^{k-\ell-1, k+\ell} \circ \mathrm{U}_{k-i, k+i+1}^{k-j-1, k+j+1}=0, \quad 1 \leqslant i \leqslant \ell-1,
\end{aligned}
$$

а при $\ell=0-$ единственное уравнение

$$
\begin{aligned}
& \left(\mathrm{id}^{\otimes(k-1)} \otimes \mathrm{b}^{\prime}\right) \circ \mathrm{U}_{k, k+1}^{k-1, k+1}\left(C^{k} \otimes C^{k+1}\right)=(-1)^{k} \mathrm{U}_{k, k}^{k-1, k}\left(C^{k} \otimes \mathrm{b}^{\prime} C^{k+1}\right)+ \\
& +\mathrm{U}_{k, k}^{k-1, k}\left(\mathrm{~b}^{\prime} C^{k+1} \otimes C^{k}\right) \text {. }
\end{aligned}
$$

При фиксированном $k$ мы применяем индукцию по $\ell$, которая в действительности является частью индукции по порядку отображений U (см. (3.21)); $\ell$ нумерует строки в (3.20). На каждом шаге мы используем и определяющие рекурсионные соотношения, и младшие (ранее доказанные) уравнения (3.32)-(3.35).

3.6.2. Начнем с базы $\ell$-индукции $\ell=0$ и покажем, что (3.35) выполнено. С учетом (3.27) уравнение (3.35) принимает вид

$$
\begin{aligned}
& \left(\mathrm{id}^{\otimes(k-1)} \otimes \mathrm{b}^{\prime}\right) \circ \mathrm{U}_{k, k+1}^{k-1, k+1}\left(C^{k} \otimes C^{k+1}\right)=-\mathrm{b}^{\prime} C^{k} \otimes \mathrm{b}^{\prime} C^{k+1}+ \\
& \quad+\left(\mathrm{id}{ }^{\otimes(k-1)} \otimes \mathrm{b}^{\prime}\right) \circ \mathrm{P}_{k, 1}\left(C^{k} \otimes \mathrm{b}^{\prime} C^{k+1}+(-1)^{k} \mathrm{~b}^{\prime} C^{k+1} \otimes C^{k}\right) .
\end{aligned}
$$

Используя очевидное тождество

$$
\left(\mathrm{id}^{\otimes(k-1)} \otimes \mathrm{b}^{\prime}\right) \circ \mathrm{P}_{k, 1}=\mathrm{P}_{k, 1} \circ\left(\mathrm{m}_{1, k+1}-\left(\mathrm{id}^{\otimes k} \otimes \mathrm{b}^{\prime}\right)\right),
$$

перепишем (3.36) в виде

$$
\begin{aligned}
& \left(\mathrm{id}^{\otimes(k-1)} \otimes \mathrm{b}^{\prime}\right) \circ \mathrm{U}_{k, k+1}^{k-1, k+1}\left(C^{k} \otimes C^{k+1}\right)= \\
& \quad-\mathrm{b}^{\prime} C^{k} \otimes \mathrm{b}^{\prime} C^{k+1}-(-1)^{k} \mathrm{P}_{k, 1}\left(\mathrm{~b}^{\prime} C^{k+1} \otimes \mathrm{b}^{\prime} C^{k}\right)+ \\
& \quad+\mathrm{P}_{k, 1} \circ \mathrm{m}_{1, k+1}\left(C^{k} \otimes \mathrm{b}^{\prime} C^{k+1}\right)+(-1)^{k} \mathrm{P}_{k, 1} \circ \mathrm{m}_{1, k+1}\left(\mathrm{~b}^{\prime} C^{k+1} \otimes C^{k}\right) .
\end{aligned}
$$


С другой стороны, отображение $\mathrm{U}_{k, k+1}^{k-1, k+1}$ выражается из рекурсионного соотношения (3.23), и поэтому (снова применяя (3.37)) можно переписать левую часть (3.38) как

$$
\begin{aligned}
& \left(\mathrm{id}^{\otimes(k-1)} \otimes \mathrm{b}^{\prime}\right) \circ \mathrm{U}_{k, k+1}^{k-1, k+1}\left(C^{k} \otimes C^{k+1}\right)= \\
& \quad=\mathrm{P}_{k, 1} \circ \mathrm{m}_{1, k+1} \circ\left(\left(\mathrm{m}_{1, k+1}-\mathrm{m}_{k+1,1}^{*}\right)\left(C^{k} \otimes C^{k+1}\right)+\right. \\
& \left.\quad+(-1)^{k}\left(\mathrm{id} \otimes \mathrm{U}_{k, k}^{k-1, k}\right) \circ \mathrm{P}_{1, k+1}\left(C^{k} \otimes C^{k+1}\right)+\left(\mathrm{id} \otimes \mathrm{U}_{k, k}^{k-1, k}\right)\left(C^{k+1} \otimes C^{k}\right)\right)- \\
& \quad-\mathrm{P}_{k, 1} \circ\left(\mathrm{id}^{\otimes k} \otimes \mathrm{b}^{\prime}\right) \circ\left(\mathrm{m}_{1, k+1}-\mathrm{m}_{k+1,1}^{*}\right)\left(C^{k} \otimes C^{k+1}\right)+\mathrm{m}_{k, k+1}\left(\mathrm{~b}^{\prime} C^{k} \otimes C^{k+1}\right)- \\
& \quad-\mathrm{b}^{\prime} C^{k} \otimes \mathrm{b}^{\prime} C^{k+1}+(-1)^{k} \mathrm{P}_{k, 1}\left(\left(\mathrm{id} \otimes \mathrm{b}^{\prime}\right) C^{k+1}\right) \otimes \mathrm{b}^{\prime} C^{k},
\end{aligned}
$$

где мы также воспользовались тем, что $\left(\right.$ id $\left.\otimes \mathrm{b}_{(k)}^{\prime}\right) \circ \mathrm{P}_{1, k+1}=\mathrm{P}_{1, k} \circ\left(\mathrm{b}_{(k)}^{\prime} \otimes \mathrm{id}\right)$ (здесь нижний индекс в $\mathrm{b}_{(k)}^{\prime}$ указывает, что $\mathrm{b}^{\prime}$ действует на $k$ тензорных множителей). Имеем теперь

$$
\begin{aligned}
& \text { левая часть (3.38) - правая часть (3.38) = } \\
& =\mathrm{P}_{k, 1} \circ \mathrm{m}_{1, k+1} \circ\left(\left(\mathrm{m}_{1, k+1}-\mathrm{m}_{k+1,1}^{*}\right)\left(C^{k} \otimes C^{k+1}\right)+\right. \\
& \text { (1) (3) } \\
& \left.+(-1)^{k}\left(\mathrm{id} \otimes \mathrm{U}_{k, k}^{k-1, k}\right) \circ \mathrm{P}_{1, k+1}\left(C^{k} \otimes C^{k+1}\right)+\left(\mathrm{id} \otimes \mathrm{U}_{k, k}^{k-1, k}\right)\left(C^{k+1} \otimes C^{k}\right)\right)- \\
& -\mathrm{P}_{k, 1} \circ\left(\mathrm{id}^{\otimes k} \otimes \mathrm{b}^{\prime}\right) \circ\left(\mathrm{m}_{1, k+1}-\mathrm{m}_{k+1,1}^{*}\right)\left(C^{k} \otimes C^{k+1}\right)+ \\
& \text { (1) (3) } \\
& +\mathrm{m}_{k \cdot k+1}\left(\mathrm{~b}^{\prime} C^{k} \otimes C^{k+1}\right)+(-1)^{k} \mathrm{P}_{k, 1} \circ \mathrm{m}_{1,2} C^{k+1} \otimes \mathrm{b}^{\prime} C^{k}- \\
& \text { (3) } \\
& \text { (2) } \\
& -\mathrm{P}_{k, 1} \circ \mathrm{m}_{1, k+1}\left(C^{k} \otimes \mathrm{b}^{\prime} C^{k+1}\right)-(-1)^{k} \mathrm{P}_{k, 1} \circ \mathrm{m}_{1, k+1}\left(\mathrm{~b}^{\prime} C^{k+1} \otimes C^{k}\right)=0,
\end{aligned}
$$

где каждая из трех групп членов (отмеченных цифрами $(1),(2)$, и (3)) обрашается в нуль по отдельности. Равенство (3.38) тем самым доказано.

3.6.3. Дальнейшие вычисления вполне непосредственны, но достаточно громоздки; мы приведем подробности только для $\ell=1$. Это представительно иллюстрирует общий случай, поскольку квадратичный член $\mathrm{U} \circ \mathrm{U}$ уже присутствует в соответствующем уравнении (3.32) (по сравнению же с (3.34) уравнения (3.32) имеют дополнительное усложнение из-за градуированной симметризации $\left.F\left(C^{k} \otimes \mathrm{b}^{\prime} C^{k+1}\right)+(-1)^{k} F\left(\mathrm{~b}^{\prime} C^{k+1} \otimes C^{k}\right)\right)$.

При $\ell=1$ два уравнения, которые следует доказать, имеют вид

$$
\begin{aligned}
& \left(\mathrm{id}^{\otimes(k-2)} \otimes \mathrm{b}^{\prime}\right) \circ \mathrm{U}_{k, k+1}^{k-2, k+2}\left(C^{k} \otimes C^{k+1}\right)+ \\
& \quad+(-1)^{k}\left(\mathrm{~b}^{\prime} \otimes \mathrm{id}^{\otimes(k+1)}\right) \circ \mathrm{U}_{k, k+1}^{k-1, k+1}\left(C^{k} \otimes C^{k+1}\right)-\mathrm{U}_{k-1, k+1}^{k-2, k+1}\left(\mathrm{~b}^{\prime} C^{k} \otimes C^{k+1}\right)+ \\
& \quad+(-1)^{k} \mathrm{U}_{k, k}^{k-2, k+1}\left(C^{k} \otimes \mathrm{b}^{\prime} C^{k+1}\right)+\mathrm{U}_{k, k}^{k-2, k+1}\left(\mathrm{~b}^{\prime} C^{k+1} \otimes C^{k}\right)= \\
& \quad \mathrm{U}_{k-1, k+1}^{k-2, k+1} \circ \mathrm{U}_{k, k+1}^{k-1, k+1}\left(C^{k} \otimes C^{k+1}\right), \\
& (-1)^{k}\left(\mathrm{id}^{\otimes(k-2)} \otimes \mathrm{b}^{\prime}\right) \circ \mathrm{U}_{k-1, k+2}^{k-2, k+2}\left(C^{k-1} \otimes C^{k+2}\right)+\mathrm{U}_{k-1, k+1}^{k-2, k+1}\left(C^{k-1} \otimes \mathrm{b}^{\prime} C^{k+2}\right)=0 .
\end{aligned}
$$


Начнем с уравнения (3.40). Вспомним рекурсионное соотношение (3.24), а также используем рекурсионное соотношение

$$
\mathrm{U}_{k-1, k+1}^{k-2, k+1}=(-1)^{k+1} \mathrm{P}_{k-1,1} \circ\left(\mathrm{m}_{2,1}^{*}-\mathrm{m}_{1,2}+\left(\mathrm{id} \otimes \mathrm{U}_{k-1, k}^{k-2, k}\right)\right) \circ \mathrm{P}_{1, k},
$$

которое следует из $(2 \boldsymbol{k}-2, \boldsymbol{k}-2)$ (т.е. из равенства $(2 \boldsymbol{k}, \boldsymbol{m})$, где мы заменили $k \rightarrow k-1$ и положили $m=k-2)$. Подставим (3.24) и (3.41) в (3.40), но сохраним $\bigcup_{k-1, k+1}^{k-2, k+1}$ "невычисленным" в комбинации id $\otimes \mathrm{U}_{k-1, k+1}^{k-2, k+1}$, возникаюшей из $(3.24)$. Как и ранее, воспользуемся тождеством (3.37) (при $k \rightarrow k-1)$. Тогда уравнение (3.40), которое следует проверить, принимает вид

$$
\begin{aligned}
(-1)^{k} \mathrm{~m}_{1, k} \circ\left(\mathrm{m}_{2,1}^{*}-\mathrm{m}_{1,2}\right) \circ \mathrm{P}_{1, k}\left(C^{k-1} \otimes C^{k+2}\right)+ \\
\quad+(-1)^{k+1}\left(\mathrm{id}^{\otimes(k-1)} \otimes \mathrm{b}^{\prime}\right) \circ\left(\mathrm{m}_{2,1}^{*}-\mathrm{m}_{1,2}\right) \circ \mathrm{P}_{1, k}\left(C^{k-1} \otimes C^{k+2}\right)+ \\
\quad+\left(\mathrm{id}^{\otimes(k-1)} \otimes \mathrm{b}^{\prime}\right) \circ\left(\mathrm{id} \otimes \mathrm{U}_{k-1, k+1}^{k-2, k+1}\right) \circ \mathrm{P}_{1, k}\left(C^{k-1} \otimes C^{k+2}\right)- \\
\quad-\mathrm{m}_{1, k} \circ\left(\mathrm{id} \otimes \mathrm{U}_{k-1, k+1}^{k-2, k+1}\right) \circ \mathrm{P}_{1, k}\left(C^{k-1} \otimes C^{k+2}\right)+ \\
\quad+(-1)^{k+1}\left(\mathrm{~m}_{2,1}^{*}-\mathrm{m}_{1,2}\right) \circ \mathrm{P}_{1, k}\left(C^{k-1} \otimes \mathrm{b}^{\prime} C^{k+2}\right)+ \\
\quad+(-1)^{k+1}\left(\mathrm{id} \otimes \mathrm{U}_{k-1, k}^{k-2, k}\right) \circ \mathrm{P}_{1, k}\left(C^{k-1} \otimes \mathrm{b}^{\prime} C^{k+2}\right)=0 .
\end{aligned}
$$

Теперь применим предположение индукции: для предшествующего отображения $\mathrm{U}_{k-1, k+1}^{k-2, k+1}$ из равенств (3.31) следует, что

$$
\left(\mathrm{id}^{\otimes(k-2)} \otimes \mathrm{b}^{\prime}\right) \circ \mathrm{U}_{k-1, k+1}^{k-2, k+1}=-(-1)^{k} \mathrm{U}_{k-1, k}^{k-2, k} \circ\left(\mathrm{id}^{\otimes(k-1)} \otimes \mathrm{b}^{\prime}\right) .
$$

Используя это вместе с простым тождеством

$$
\begin{gathered}
\left(\mathrm{id}^{\otimes(k-1)} \otimes \mathrm{b}^{\prime}\right) \circ\left(\mathrm{m}_{2,1}^{*}-\mathrm{m}_{1,2}\right)+\left(\mathrm{m}_{1, k}-\mathrm{m}_{k, 1}^{*}\right) \circ\left(\mathrm{id}^{\otimes(k-1)} \otimes \mathrm{b}^{\prime}\right) \circ \mathrm{P}_{k, 1}- \\
-\mathrm{m}_{1, k} \circ\left(\mathrm{m}_{2,1}^{*}-\mathrm{m}_{1,2}\right)=\mathrm{m}_{1, k} \circ \mathrm{m}_{1,2}-\mathrm{m}_{1,2} \circ \mathrm{m}_{1, k+1},
\end{gathered}
$$

приводим (3.42) к виду

$$
\begin{aligned}
& (-1)^{k+1}\left(\mathrm{~m}_{1, k} \circ \mathrm{m}_{1,2}-\mathrm{m}_{1,2} \circ \mathrm{m}_{1, k+1}\right)- \\
& \quad-\mathrm{m}_{1, k} \circ\left(\mathrm{id} \otimes \mathrm{U}_{k-1, k+1}^{k-2, k+1}\right)-(-1)^{k}\left(\mathrm{id} \otimes \mathrm{U}_{k-1, k}^{k-2, k}\right) \circ \mathrm{m}_{1, k+1}=0 .
\end{aligned}
$$

Записывая $\mathrm{U}_{k-1, k+1}^{k-2, k+1}=\mathrm{P}_{k-1,1} \circ \widetilde{\mathrm{U}}$, имеем $\mathrm{m}_{1, k} \circ\left(\right.$ id $\left.\otimes \mathrm{U}_{k-1, k+1}^{k-2, k+1}\right)=\mathrm{m}_{1,2} \circ($ id $\otimes \widetilde{\mathbf{U}})$; вспоминая из (3.41), как на самом деле выглядит $\widetilde{U}$, видим, что равенство (3.44) удовлетворяется тождественно (независимо от каких-либо свойств $\mathrm{U}_{k-1, k}^{k-2, k}$ ). Таким образом, равенство (3.40) доказано.

Докажем далее равенство (3.39). В его левой части $\mathrm{U}_{k, k+1}^{k-2, k+2}$ выражается из рекурсионного соотношения (3.25). Выразим также $\mathrm{U}_{k, k+1}^{k-1, k+1}$, используя равенство (3.23), 
за исключением тех $\mathrm{U}_{k, k+1}^{k-1, k+1}$, которые возникают из (3.25); для последних используем уравнение (3.35) с тем, чтобы переписать возникающую таким образом комбинацию $\left(\mathrm{id}^{\otimes(k-1)} \otimes \mathrm{b}^{\prime}\right) \circ \mathrm{U}_{k, k+1}^{k-1, k+1}$ как

$$
\begin{aligned}
\left(\mathrm{id}^{\otimes(k-1)} \otimes \mathrm{b}^{\prime}\right) \circ \mathrm{U}_{k, k+1}^{k-1, k+1}\left(C^{k} \otimes C^{k+1}\right)= & (-1)^{k} \mathrm{U}_{k, k}^{k-1, k}\left(C^{k} \otimes \mathrm{b}^{\prime} C^{k+1}\right)+ \\
& +\mathrm{U}_{k, k}^{k-1, k}\left(\mathrm{~b}^{\prime} C^{k+1} \otimes C^{k}\right) .
\end{aligned}
$$

В правой части (3.39) используем рекурсионные соотношения (3.23) и (3.41) и затем применим предположение индукции, из которого следует уравнение

$$
\begin{gathered}
(-1)^{k} \mathrm{U}_{k-1, k}^{k-2, k}\left(\mathrm{~b}^{\prime} C^{k} \otimes C^{k}\right)+\mathrm{U}_{k-1, k}^{k-2, k} \circ \mathrm{U}_{k, k}^{k-1, k}\left(C^{k} \otimes C^{k}\right)+ \\
+(-1)^{k}\left(\mathrm{id}^{\otimes(k-2)} \otimes \mathrm{b}_{(k+1)}^{\prime}\right) \circ \mathrm{U}_{k, k}^{k-2, k+1}\left(C^{k} \otimes C^{k}\right)+ \\
+\left(\mathrm{b}_{(k-1)}^{\prime} \otimes \mathrm{id}^{\otimes k}\right) \circ \mathrm{U}_{k, k}^{k-1, k}\left(C^{k} \otimes C^{k}\right)=0 .
\end{gathered}
$$

Используем его для исключения билинейного члена по U. Непосредственные манипуляции с учетом (3.37) и очевидного тождества

$$
\left(\mathrm{b}_{(k-1)}^{\prime} \otimes \mathrm{id}^{\otimes(k+1)}\right) \circ \mathrm{P}_{k, 1}=\mathrm{P}_{k-1,1} \circ\left(\mathrm{id} \otimes \mathrm{b}_{(k-1)}^{\prime} \otimes \mathrm{id}^{\otimes k}\right)
$$

показывают, что слагаемые, возникающие из двух последних членов (3.45), сокрашаются с подобными членами из левой части (3.39). Далее, используя в левой части (3.39) рекурсионные соотношения для $\mathrm{U}_{k-1, k+1}^{k-2, k+1}$ и $\mathrm{U}_{k, k}^{k-2, k+1}$, получаем в соответствии с $(3.41)$, что

$$
\begin{gathered}
-\mathrm{P}_{1, k-1} \circ \mathrm{U}_{k-1, k+1}^{k-2, k+1}\left(\mathrm{~b}^{\prime} C^{k} \otimes C^{k+1}\right)=(-1)^{k}\left(\mathrm{~m}_{1, k}-\mathrm{m}_{k, 1}^{*}\right)\left(\mathrm{b}^{\prime} C^{k} \otimes C^{k+1}\right)+ \\
+(-1)^{k}\left(\mathrm{id} \otimes \mathrm{U}_{k-1, k}^{k-2, k}\right) \circ \mathrm{P}_{1, k}\left(\mathrm{~b}^{\prime} C^{k} \otimes C^{k+1}\right) .
\end{gathered}
$$

Наконец, учитьвая рекурсионное соотношение

$\mathrm{U}_{k, k}^{k-2, k+1}\left(C^{k} \otimes C^{k}\right)=-\mathrm{P}_{k-1,1} \circ\left(\mathrm{id} \otimes \mathrm{U}_{k-1, k}^{k-2, k}\right)\left(C^{k} \otimes C^{k}\right)-(-1)^{k} \mathrm{P}_{k-1,1} \circ \mathrm{U}_{k, k}^{k-1, k}\left(C^{k} \otimes C^{k}\right)$

(являюшееся равенством (3.29) при $k \rightarrow k-1$ и $m=k-2$ ), используя тождество

$$
\begin{gathered}
(-1)^{k}\left(\mathrm{id} \otimes \mathrm{b}_{(k-1)}^{\prime} \otimes \mathrm{id}^{\otimes k}\right) \circ\left(\mathrm{m}_{1, k+1}-\mathrm{m}_{k+1,1}^{*}\right)\left(C^{k} \otimes C^{k+1}\right)= \\
=(-1)^{k}\left(\mathrm{~m}_{1, k}-\mathrm{m}_{k, 1}^{*}\right)\left(\left(\left(\mathrm{id} \otimes \mathrm{b}^{\prime}\right) C^{k}\right) \otimes C^{k+1}\right)
\end{gathered}
$$

и применяя вытекающее из предположения индукции равенство (3.43) к комбинации $\left(\mathrm{id}^{\otimes(k-1)} \otimes \mathrm{b}^{\prime}\right) \circ\left(\mathrm{id} \otimes \mathrm{U}_{k-1, k+1}^{k-2, k+1}\right)$, перепишем (3.39) в виде

$$
\begin{aligned}
& \mathrm{m}_{1, k} \circ\left(\left(\mathrm{id} \otimes \mathrm{U}_{k-1, k+1}^{k-2, k+1}\right)\left(C^{k} \otimes C^{k+1}\right)-\left(\mathrm{id} \otimes \mathrm{U}_{k, k}^{k-2, k+1}\right)\left(C^{k+1} \otimes C^{k}\right)-\right. \\
& \left.-(-1)^{k}\left(\mathrm{id} \otimes \mathrm{U}_{k, k}^{k-2, k+1}\right) \circ \mathrm{P}_{1, k+1}\left(C^{k} \otimes C^{k+1}\right)\right)+ \\
& +\underline{(-1)^{k}\left(\mathrm{~m}_{1, k}-\mathrm{m}_{k, 1}^{*}\right)\left(\mathrm{m}_{1,2} C^{k} \otimes C^{k+1}\right)}= \\
& =(-1)^{k} \mathrm{~m}_{1,2} \circ \underline{\left(\mathrm{m}_{1, k+1}-\mathrm{m}_{k+1,1}^{*}\right)\left(C^{k} \otimes C^{k+1}\right)}+\left(\mathrm{id} \otimes \mathrm{U}_{k, k}^{k-1, k}\right)\left(C^{k+1} \otimes C^{k}\right)+ \\
& \left.+(-1)^{k}\left(\mathrm{id} \otimes \mathrm{U}_{k, k}^{k-1, k}\right) \circ \mathrm{P}_{1, k+1}\left(C^{k} \otimes C^{k+1}\right)\right)+ \\
& +(-1)^{k+1}\left(\mathrm{id} \otimes \mathrm{U}_{k-1, k}^{k-2, k}\right) \circ\left(\mathrm{m}_{1, k+1}-\mathrm{m}_{k+1,1}^{*}\right)\left(C^{k} \otimes C^{k+1}\right)+ \\
& +\left(\mathrm{id} \otimes \mathrm{U}_{k-1, k}^{k-2, k}\right) \circ \mathrm{m}_{1,2}\left(C^{k+1} \otimes C^{k}\right) \text {. }
\end{aligned}
$$


Очевидно, что подчеркнутые члены можно заменить на

$$
(-1)^{k}\left(\mathrm{~m}_{1, k} \circ \mathrm{m}_{1,2}-\mathrm{m}_{1,2} \circ \mathrm{m}_{1, k+1}\right)
$$

в левой части. Далее, рекурсионное соотношение (3.41) позволяет вычислить

$$
\begin{aligned}
\mathrm{m}_{1, k} & \circ\left(\mathrm{id} \otimes \mathrm{U}_{k-1, k+1}^{k-2, k+1}\right)= \\
& =(-1)^{k+1} \mathrm{~m}_{1, k} \circ\left(\mathrm{id} \otimes\left(\mathrm{P}_{k-1,1} \circ\left(\mathrm{m}_{2,1}^{*}-\mathrm{m}_{1,2}+\left(\mathrm{id} \otimes \mathrm{U}_{k-1, k}^{k-2, k}\right)\right) \circ \mathrm{P}_{1, k}\right)\right)= \\
& =(-1)^{k+1} \mathrm{~m}_{1, k} \circ \mathrm{P}_{k, 2} \circ\left(\mathrm{m}_{3,2}^{*}-\mathrm{m}_{2,3}+\left(\mathrm{id}^{\otimes 2} \otimes \mathrm{U}_{k-1, k}^{k-2, k}\right)\right) \circ \mathrm{P}_{2, k+1}= \\
& =(-1)^{k+1}\left(\mathrm{~m}_{1, k} \circ \mathrm{m}_{1,2}-\mathrm{m}_{1,2} \circ \mathrm{m}_{1, k+1}\right)+(-1)^{k+1}\left(\mathrm{id} \otimes \mathrm{U}_{k-1, k}^{k-2, k}\right) \circ \mathrm{m}_{1, k+1} .
\end{aligned}
$$

Как и ранее, эта выкладка не использует никаких свойств отображения $\mathrm{U}_{k-1, k}^{k-2, k}$ (см. рисунок, иллюстрируюший преобразование слагаемого, включаюшего $\mathrm{U}_{k-1, k}^{k-2, k}$; слагаемые, содержащие $\mathrm{m}_{3,2}^{*}$ и $\mathrm{m}_{2,3}$, преобразуются аналогично). Последнее слагаемое сокращается с подобным в правой части (3.48), члены $\mathrm{m} \circ \mathrm{m}$ сокращают таковые в (3.49), и равенство (3.48) принимает вид

$$
\begin{aligned}
-\mathrm{m}_{1, k} & \circ\left(\left(\mathrm{id} \otimes \mathrm{U}_{k, k}^{k-2, k+1}\right)\left(C^{k+1} \otimes C^{k}\right)+\right. \\
& \left.+(-1)^{k}\left(\mathrm{id} \otimes \mathrm{U}_{k, k}^{k-2, k+1}\right) \circ \mathrm{P}_{1, k+1}\left(C^{k} \otimes C^{k+1}\right)\right)= \\
= & \mathrm{m}_{1,2} \circ\left((-1)^{k}\left(\mathrm{id} \otimes \mathrm{U}_{k, k}^{k-1, k}\right)\left(C^{k+1} \otimes C^{k}\right)+\right. \\
& \left.+\left(\mathrm{id} \otimes \mathrm{U}_{k, k}^{k-1, k}\right) \circ \mathrm{P}_{1, k+1}\left(C^{k} \otimes C^{k+1}\right)\right)+ \\
& +(-1)^{k}\left(\mathrm{id} \otimes \mathrm{U}_{k-1, k}^{k-2, k}\right) \circ \mathrm{m}_{k+1,1}^{*}\left(C^{k} \otimes C^{k+1}\right)+ \\
& +\left(\mathrm{id} \otimes \mathrm{U}_{k-1, k}^{k-2, k}\right) \circ \mathrm{m}_{1,2}\left(C^{k+1} \otimes C^{k}\right) .
\end{aligned}
$$

Используя рекурсионное соотношение (3.47) и применяя рассуждения, полностью аналогичные тем, что применялись к (3.50), легко видеть, что это равенство является тождеством. Уравнение (3.39) доказано.

3.7. Окончание доказательства теоремы 3.1. Остается доказать равенство $(2.16)$, или $X^{(3)}=0$, для БРСТ-дифференциала $\Omega$. Доказательство опирается на алгебраические следствия уже установленных уравнений (2.15). Некоторые подробности таковы. Запишем $\Omega$ как в $(2.12)$ в случае, когда $\tau_{A}$ и $U_{A B}$ (с суммированием по повторяющимся индексам) имеют вид

$$
\tau_{A}=t_{A}+Z_{A}^{B} \overline{\mathcal{P}}_{B}, \quad U_{A B}=U_{A B}^{C} \overline{\mathcal{P}}_{C}+\frac{1}{2}(-1)^{\varepsilon_{C}} U_{A B}^{C D} \overline{\mathcal{P}}_{D} \overline{\mathcal{P}}_{C}
$$

с не зависящими от $\overline{\mathcal{P}}$ и $C$ величинами $t_{A}, Z_{A}^{B}, U_{A B}^{C}$ и $U_{A B}^{C D}$. Здесь $\varepsilon\left(C^{A}\right)=\varepsilon\left(\overline{\mathcal{P}}_{A}\right)=$ $\varepsilon_{A}+1$. Вообще говоря, $\mathbb{Z}_{2}$-градуировки равны $\varepsilon\left(t_{A}\right)=\varepsilon_{A}, \quad \varepsilon\left(Z_{A}^{B}\right)=\varepsilon_{A}+\varepsilon_{B}+1$, $\varepsilon\left(U_{A B}^{C}\right)=\varepsilon_{A}+\varepsilon_{B}+\varepsilon_{C}$ и $\varepsilon\left(U_{A B}^{C D}\right)=\varepsilon_{A}+\varepsilon_{B}+\varepsilon_{C}+\varepsilon_{D}+1$, но в нашем конкретном случае все эти отображения являются четными. Разлагая $(2.14)-(2.16)$ по духовым 


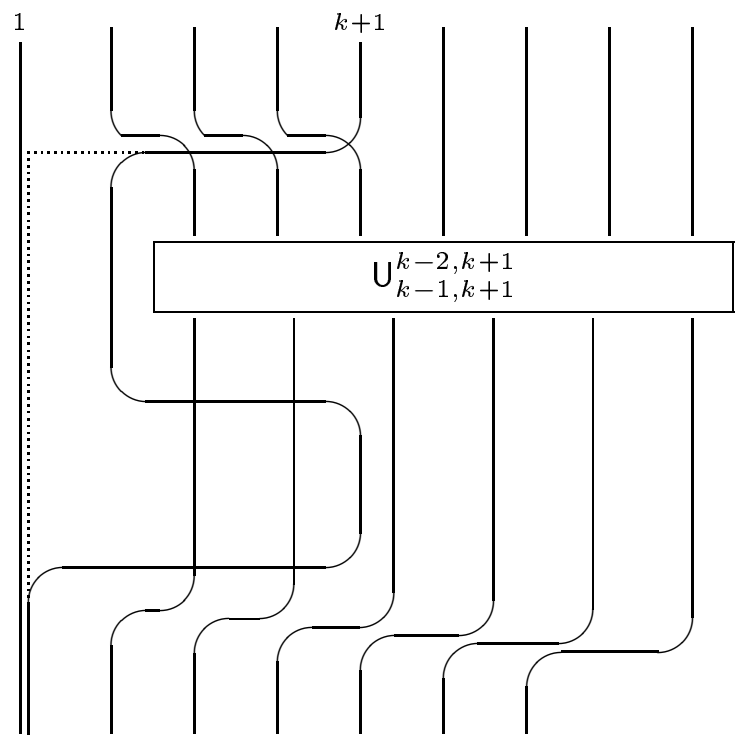

Графичиеское представление $\mathrm{m}_{1, k} \circ \mathrm{P}_{k, 2} \circ\left(\mathrm{id}^{\otimes 2} \otimes \mathrm{U}_{k-1, k}^{k-2, k}\right) \circ \mathrm{P}_{2, k+1}$ (изображенное при $k=4)$, показывающее, что это выражение тождественно совпадает с $\left(\mathrm{id} \otimes \mathrm{U}_{k-1, k}^{k-2, k}\right)$ 。 $\mathrm{m}_{1, k+1}$ (отмеченное пунктиром положение линии, изображающей $(k+1)$ )-й тензорный множитель). Операторы $\mathrm{P}_{k, 2}$ и $\mathrm{m}_{1, k}$ изображаются сплетением и слиянием линий, представляющих соответствующие тензорные множители. Порядок применения операций справа налево здесь соответствует порядку сверху вниз.

импульсам, помимо основных соотношений $Z_{A}^{B} t_{B}=0, Z_{A}^{B} Z_{B}^{C}=0$ и $\left[t_{A}, t_{B}\right]=U_{A B}^{C} t_{C}$ получаем коммутаторы, включаюшие $Z_{A}^{B}$ :

$$
\begin{aligned}
& {\left[t_{A}, Z_{B}^{D}\right]-(-1)^{\varepsilon_{A} \varepsilon_{B}}\left[t_{B}, Z_{A}^{D}\right]=} \\
& =U_{A B}^{C} Z_{C}^{D}-U_{A B}^{D C} t_{C}-(-1)^{\varepsilon_{B}} Z_{A}^{C} U_{C B}^{D}+(-1)^{\varepsilon_{A}\left(1+\varepsilon_{B}\right)} Z_{B}^{C} U_{C A}^{D}+\frac{1}{2} U_{A B}^{E C} U_{C E}^{D} \\
& (-1)^{\left(\varepsilon_{B}+1\right)\left(\varepsilon_{D}+1\right)}\left[Z_{A}^{D}, Z_{B}^{E}\right]-(-1)^{\left(\varepsilon_{B}+1\right)\left(\varepsilon_{E}+1\right)+\varepsilon_{D} \varepsilon_{E}}\left[Z_{A}^{E}, Z_{B}^{D}\right]=-\frac{1}{2} U_{A B}^{F C} U_{C F}^{D E}+ \\
& \quad+(-1)^{\varepsilon_{D}} U_{A B}^{D C} Z_{C}^{E}-(-1)^{\varepsilon_{E}\left(1+\varepsilon_{D}\right)} U_{A B}^{E C} Z_{C}^{D}+ \\
& \quad+(-1)^{\varepsilon_{B}} Z_{A}^{C} U_{C B}^{D E}-(-1)^{\varepsilon_{A}\left(1+\varepsilon_{B}\right)} Z_{B}^{C} U_{C A}^{D E}
\end{aligned}
$$

а также (из (2.16)) тождество Якоби

$$
(-1)^{\varepsilon_{A} \varepsilon_{C}} U_{A B}^{D} U_{D C}^{E}+\operatorname{cycle}(A, B, C)=0
$$

и его обобшения, включаюшие $U_{A B}^{C D}$ с четырьмя индексами:

$$
\begin{aligned}
& (-1)^{\varepsilon_{A} \varepsilon_{C}}\left(U_{A B}^{D} U_{D C}^{E F}+(-1)^{\left(\varepsilon_{E}+1\right) \varepsilon_{C}} U_{A B}^{E D} U_{D C}^{F}-\right. \\
& \left.-(-1)^{\left(\varepsilon_{F}+1\right) \varepsilon_{C}+\varepsilon_{E} \varepsilon_{F}} U_{A B}^{F D} U_{D C}^{E}\right)+\operatorname{cycle}(A, B, C)=0 \\
& (-1)^{\left(\varepsilon_{E}+1\right)\left(\varepsilon_{C}+1\right)+\varepsilon_{A} \varepsilon_{C}+\varepsilon_{E} \varepsilon_{G}} U_{A B}^{E D} U_{D C}^{F G}+ \\
& \quad+\operatorname{cycle}(A, B, C)+\operatorname{cycle}(E, F, G)=0 .
\end{aligned}
$$


Доказательство в п. 3.5, 3.6 есть не что иное, как демонстрация равенств (3.52) и (3.53). Остаюшиеся уравнения (3.54)-(3.56) получаются как алгебраические условия согласованности равенств (3.52) и (3.53) с использованием конкретных свойств $Z_{A}^{B}$ и “правил отбора" для $U_{A B}^{C}$ и $U_{A B}^{C D}$. Для этого вычислим левую часть тождества Якоби $(-1)^{\varepsilon_{A} \varepsilon_{C}}\left[\left[\tau_{A}, \tau_{B}\right], \tau_{C}\right]+\operatorname{cycle}(A, B, C)=0$. Двойной коммутатор содержит члены нулевой, первой и второй степени по духовым импульсам,

$$
\left[\left[\tau_{A}, \tau_{B}\right], \tau_{C}\right]=J_{A B C}+J_{A B C}^{H} \overline{\mathcal{P}}_{H}+J_{A B C}^{H G} \overline{\mathcal{P}}_{G} \overline{\mathcal{P}}_{H},
$$

где $J_{A \text { В }}=U_{A B}^{D} U_{D C}^{E} t_{E}$ и

$$
\begin{aligned}
& J_{A B C}^{H}=-(-1)^{\varepsilon_{C}} U_{A B}^{D} Z_{D}^{E} U_{E C}^{H}+(-1)^{\varepsilon_{D}\left(\varepsilon_{C}+1\right)} U_{A B}^{D} Z_{C}^{E} U_{E D}^{H}+U_{A B}^{D} U_{D C}^{E} Z_{E}^{H}+ \\
& +\frac{1}{2} U_{A B}^{D} U_{D C}^{E K} U_{K E}^{H}+(-1)^{\varepsilon_{E} \varepsilon_{H}} U_{A B}^{D} U_{D C}^{E H} t_{E}+ \\
& +(-1)^{\varepsilon_{D}+\left(\varepsilon_{H}+1\right)\left(\varepsilon_{D}+\varepsilon_{C}\right)} U_{A B}^{D H} U_{D C}^{E} t_{E}+ \\
& +(-1)^{\varepsilon_{A} \varepsilon_{C}+\varepsilon_{B} \varepsilon_{C}+\varepsilon_{B}}\left[t_{C}, Z_{A}^{D}\right] U_{D B}^{H}-(-1)^{\varepsilon_{A} \varepsilon_{B}+\varepsilon_{B} \varepsilon_{C}+\varepsilon_{A} \varepsilon_{C}+\varepsilon_{A}}\left[t_{C}, Z_{B}^{D}\right] U_{D A}^{H}
\end{aligned}
$$

(выражение для $J_{A B C}^{H G}$ получается непосредственно, но оказывается достаточно громоздким). Обрашение в нуль циклической суммы от $(-1)^{\varepsilon_{A} \varepsilon_{C}} J_{A B C}$ не позволяет сразу заключить, что циклическая сумма от $(-1)^{\varepsilon_{A} \varepsilon_{C}} U_{A B}^{D} U_{D C}^{E}$ обращается в нуль, поскольку, как видно из сравнения (3.13) и первого из равенств $(3.51), t_{A}$-члены присутствуют в $\tau_{A}$ только в “неприводимом секторе", при $A=a$, в каковом случае $t_{a}$ являются элементами базиса в $\mathcal{A}$. Следует поэтому различать случаи, когда индексы $A, B, C$ принимают "младшие" и “старшие" значения, т.е. нумеруют соответственно элементы базиса в $\mathcal{A}$ и элементы базисов в $\mathcal{A}^{\otimes n}$ при $n \geqslant 2$. Но когда все три индекса принимают "старшие" значения, члены с коммутаторами в формуле для $J_{A B C}^{H}$ обрашаются в нуль. Более того, правила отбора для отображений $\mathrm{U}$ (а именно требование, чтобы значение $\mathrm{U}_{m, n}^{m^{\prime}, n^{\prime}}$ было ненулевым только при $\left.m+n=m^{\prime}+n^{\prime}+1\right)$ показывают, что члены, содержащие $t_{E}$, также можно отбросить при общих "старших" значениях индексов $A, B$, $C$. Среди остаюшихся в $J_{A B C}^{H}$ четырех слагаемых одно пропорционально единичному элементу алгебры, тогда как три других не пропорциональны (при вычислении на элементе общего положения в соответствующих тензорных степенях алгебры $\mathcal{A}$ ). К этим трем элементам (занимающим первую строчку в последней формуле) мы применим тот же прием взятия фактора по $\mathbb{C} \cdot 1$, что и в п. 3.5.3. В используемой нормировке это сводится к замене $Z_{A}^{B}$ на $(-1)^{\varepsilon} A \delta_{A}^{B}$. Два из трех слагаемых тогда сокрашают друг друга, а циклическая сумма остаюшегося дает требуемое соотношение (3.54); остальные соотношения доказываются аналогично.

\section{8. Дополнительные замечания.}

3.8.1. "Гамильтонианы" и когомологии. Задачу о когомологиях дифференциала $\boldsymbol{\Omega}$ можно в принципе рассматривать на различных пространствах. Как и в отношении общего вида $\boldsymbol{\Omega}$, мы позаимствуем одну постановку такой задачи из квантования калибровочных теорий. В настоящих калибровочных теориях (квантовый) гамильтониан $\mathcal{H} \in \mathbb{A}_{\text {gh }}^{\hbar}$ коммутирует с $\boldsymbol{\Omega}$ (и потому естественным образом рассматривается по модулю $\Omega$-точных членов). Физическое требование состоит в том, чтобы $\mathrm{gh} \mathcal{H}=0$. В калибровочных теориях с замкнутой алгеброй гамильтонианы, как правило, не более чем 
линейны по духам $C$, но могут зависеть от духовых импульсов. Мы ограничимся случаем не более чем билинейной зависимости от духовых импульсов и, кроме того, выберем часть, зависяшую от духовых импульсов, “скалярной” в смысле коммутирования с элементами из $\mathbb{A}^{\hbar}$ ("исходными" операторами теории, не включаюшими духи). Такие гамильтонианы имеют вид

$$
\mathcal{H}=H+\sum_{r \geqslant 1}\left\langle\mathrm{~V}_{r}\left(C^{r}\right), P_{r}\right\rangle+\sum_{s>r \geqslant 1} \sum_{r+s}\left\langle\mathrm{~V}_{r+s}^{r, s}\left(C^{r+s}\right), P_{r} \otimes P_{s}\right\rangle,
$$

где $H \in \mathcal{A}, \mathrm{V}_{r} \in \operatorname{Hom}\left(\mathcal{A}^{\otimes r}, \mathcal{A}^{\otimes r}\right)$ и $\mathrm{V}_{m}^{r, s} \in \operatorname{Hom}\left(\mathcal{A}^{\otimes m}, \mathcal{A}^{\otimes r} \otimes \mathcal{A}^{\otimes s}\right)$. Линейные по духам $C$ члены в коммутаторе имеют вид

$$
\begin{aligned}
{\left.[\mathcal{H}, \boldsymbol{\Omega}]\right|_{C^{\otimes 1}}=} & \left.H, C^{1}\right]+\mathrm{V}_{1}\left(C^{1}\right)+\sum_{s \geqslant 1} \iota\left(\operatorname{ad}_{1} \cdot \mathrm{Z}^{s+1}\left(C^{s+1}\right)\right)_{P_{s}}+ \\
& +\sum_{s \geqslant 2}(-1)^{s} \iota\left(\mathrm{V}_{s+1}^{1, s}\left(C^{s+1}\right)\right)_{P_{s}}+\sum_{s \geqslant 1}(-1)^{s} \iota\left(\mathrm{Z}^{s+1} \circ \mathrm{V}_{s+1}\left(C^{s+1}\right)\right)_{P_{s}}+ \\
& +\sum_{s \geqslant 1}(-1)^{s} \iota\left(\left(\mathrm{id} \otimes \mathrm{V}_{s}\right) \circ \mathrm{Z}^{s+1}\left(C^{s+1}\right)\right)_{P_{s}}+ \\
& +\sum_{s>r \geqslant 1} \sum_{r=1)^{r s+r+s}\left\langle\mathrm{U}_{r, s}^{r+s-1} \circ \mathrm{V}_{r+s}^{r, s}\left(C^{r+s}\right), P_{r+s-1}\right\rangle+\cdots}
\end{aligned}
$$

где многоточием обозначены пропорииональные $\mathrm{V}_{r+s}^{r, s}$ членьl, квадратичные по импульсам.

Для любого $H \in \mathcal{A}$ существуют отображения $\mathrm{V}_{r}$ такие, что $[\mathcal{H}, \boldsymbol{\Omega}]=0$. Действительно, обращение в нуль не зависящих от $P_{n}$ членов в коммутаторе выражается как

$$
\left[H, C^{1}\right]+\mathrm{V}_{1}\left(C^{1}\right)=0
$$

а обрашение в нуль линейных по $P_{n}$ слагаемых - как

$$
\operatorname{ad}_{H} \cdot \mathrm{Z}^{n+1}+(-1)^{n} \mathrm{Z}^{n+1} \circ \mathrm{V}_{n+1}+(-1)^{n}\left(\mathrm{id} \otimes \mathrm{V}_{n}\right) \circ \mathrm{Z}^{n+1}=0
$$

что немедленно дает решение

$$
\mathrm{V}_{n}=(-1)^{n} \mathrm{~L}_{H}
$$

Остается заметить, что $\mathrm{L}_{H} \circ \mathrm{U}_{m, n}^{\ell, m+n-1-\ell}-\mathrm{U}_{m, n}^{\ell, m+n-1-\ell} \circ \mathrm{L}_{H}=0$ для любого $H \in \mathcal{A}$. Более того, гамильтониан $\mathcal{H}$ указанного вида не является $\Omega$-точным, и поэтому когомологии $\boldsymbol{\Omega}$ на пространстве таких операторов содержат алгебру $\mathcal{A}$.

3.8.2. Автоморфизмы. Очевидно, что соотношения $\boldsymbol{\Omega} \boldsymbol{\Omega}=0$ сохраняются при преобразованиях подобия $\boldsymbol{\Omega} \mapsto \mathfrak{U} \Omega \mathfrak{U}^{-1}$, где $\mathfrak{U}$ - произвольный обратимый оператор на barрезольвенте; естественный подкласс таких операторов дается "внутренними" автоморфизмами, $\mathfrak{U}=e^{\mathfrak{G}}$ (в предположении, что экспоненциальное отображение сушествует), где $\mathfrak{G}, \operatorname{gh} \mathfrak{G}=0,-$ произвольный оператор на bar-резольвенте. Следуя логике, использованной выше, можно дополнительно ограничить класс преобразований требованием, 
чтобы все коэффициенты оператора $\mathfrak{G}$ за исключением постоянного члена были скалярными (но даже при таких операторах явный вид преобразованного БРСТ-дифференциала не будет соответствовать замкнутой алгебре). Этот класс содержит, в частности, преобразования, описанные в работе [11], которые "ковариантно" действуют на умножение $\mathrm{m} \in \operatorname{Hom}(\mathcal{A} \otimes \mathcal{A}, \mathcal{A})$ и тем самым "сохраняют ассоциативность". При ограничении на преобразования, сохраняюшие максимальные степени в разложении по духам и духовым импульсам, может оказаться возможным получить такие решения для отображений U, что выписанные в (3.22) отображения не обязательно обрашаются в нуль.

Благодарности. Авторы признательны Б. Л. Фейгину, М. А. Григорьеву и И. В. Тютину за полезные обсуждения. Работа частично поддержана РФФИ (гранты № 02-01-00930 и № 00-15-96566), Фондом содействия российской науке и INTAS (грант № 00-00262).

\section{Список литературы}

[1] I. A. Batalin, G. A. Vilkovisky. Phys. Lett. B. 1981. V. 102. P. $27-31$.

[2] I. A. Batalin, E. S. Fradkin. Ann. Inst. H. Poincaré (Phys. Theor.). 1988. V. 49. P. 145-214.

[3] M. Henneaux, C. Teitelboim. Quantization of gauge systems. Princeton: Princeton University Press, 1992.

[4] J. Stasheff . Homological reduct ion of constrained Poisson algebras. q-alg/9603021.

[5] I. A. Batalin, E.S. Fradkin. Phys. Lett. B. 1983. V. 122. P. 157-164.

[6] I. A. Batalin, G. A. Vilkovisky. Phys. Lett. B. 1983. V. 120. P. 166-170; Phys. Rev. D. 1983. V. 28. P. 2567-2582; Erratum. 1984. V. 30. P. 508; G. A. Vilkovisky. Lett. Math. Phys. 1999. V. 49. P. $123-130$.

[7] R. Kallosh, W. Troost, A. Van Proeyen. Phys. Lett. B. 1988. V. 212. P. 428-436; E. Bergshoeff, R. Kallosh, T. Ortin, G. Papadopoulos. Nucl. Phys. B. 1997. V. 502. P. $149-169$.

[8] F. Bayen, M. Flato, C. Fronsdal, A. Lichnerowicz, D. Sternheimer. Ann. Phys. 1978. V. 111. P. 61-110; P. 111-151; B. V. Fedosov. J. Diff. Geom. 1994. V. 40. P. 213-238; Deformation quantization and index theory. Berlin: Akademie-Verl., 1996 (Mathematical topics: 9); M. Kontsevich. Deformation quantization of Poisson manifolds. I. q-alg/9709040.

[9] W. Arveson. The harmonic analysis of automorphism groups. In: Operator Algebras and Applications. Part I. Proc. of the 28th Summer Institute of the American Mathematical Society Held at Queen's Univ., Kingston, Ont., July 14-August 2, 1980. Symp. Pure Math. V. 38. Ed. R. V. Kadison. Providence, RI: AMS, 1982. P. 199-269; M. Karoubi. Astérisque. 1987. V. 149. P. 1-147.

[10] J. Cuntz, D. Quillen. J. Am. Math. Soc. 1995. V. 8. P. 251-289.

[11] I. A. Batalin, I. V. Tyutin. J. Math. Phys. 1993. V. 34. P. 369-380. 